\title{
Exterior Complex Scaling and the AC-Stark Effect in a Coulomb Field
}

\author{
Sandro Graffi ${ }^{\star}$ and Kenji Yajima ${ }^{\star \star}$ \\ Department of Mathematics, Princeton University, Princeton, NJ 08544, USA
}

\begin{abstract}
By means of the exterior complex scaling of B. Simon an existence proof of resonances is given for the time-dependent Schrödinger equation

$$
i \frac{\partial \psi}{\partial t}=\left(-\Delta+V+\mu x_{1} \cos \omega t\right) \psi
$$

where $V$ belongs to a class of potentials which includes the Coulomb one. The resonance width is given by the Fermi Golden Rule to second order perturbation theory and is nonzero for $\mu$ small and almost every $\omega$.
\end{abstract}

\section{Introduction}

The time dependent Schrödinger equation

$$
i \frac{\partial \psi}{\partial t}=\left(-\Delta+V(x)+\frac{1}{2} \mu x_{1} \cos \omega t\right) \psi
$$

describes the so-called AC-Stark effect, i.e. the quantum motion of a particle of mass $\frac{1}{2}$, unit charge, coordinates $x=\left(x_{1}, x_{2}, x_{3}\right) \in \mathbb{R}^{3}$ in a potential field $V(x)$ under the action of an oscillating electric field of strength $\frac{1}{2} \mu \cos \omega t, \mu \in \mathbb{R}$, directed along the $x_{1}$ axis. Here $\omega \in \mathbb{R}_{+}$, and $\hbar=1$. If $V$ is not only dilation analytic, but also satisfies some extra smoothness assumptions, Yajima [12] has obtained, through a synthesis of Floquet theory and complex scaling, a mathematical justification of the well-known physical picture associated with the time dependent perturbation problem (1.1) (see e.g. $[5,11]$ ).

Let us describe in some detail the results of [12], because the purpose of this paper is to extend the main one, i.e. the existence of resonances, to a class of potentials sufficiently general to include the Coulomb one. Setting, as in [3]:

$$
\begin{gathered}
\psi(t, x)=T(t) v(t, x), \\
(T(t) f)(x)=e^{-i\left(\omega^{-1} \mu x_{1} \sin \omega t-16 \mu^{-2} \omega^{-3} \sin 2 \omega t+8 \mu^{2} \omega^{-2} t\right)} f\left(x_{1}-\mu \omega^{-2} \cos \omega t, x_{2} x_{3}\right),
\end{gathered}
$$

* Present address : Istituto di Matematica, Università di Bologna, I-40127 Bologna, Italy

$\star \star$ Partially supported by NSF Grant No. MCS-80-23339-A01. Address since September 1, 1982: Department of Pure and Applied Science, University of Tokyo, Komaba, Meguro-Ku, Tokyo, Japan 
Equation (1.1) becomes :

$$
i \frac{\partial v}{\partial t}=\left(-\Delta+V\left(x_{1}+\omega^{-2} \mu \cos \omega t, x_{2}, x_{3}\right)\right) v \equiv H(t, \mu) v .
$$

Then Eq. (1.3) generates a unitary propagator $U(t, s ; \mu)$ and the following assertions hold true:

(1) The eigenvalues of the Floquet operator $U\left(s+\frac{2 \pi}{\omega}, s ; \mu\right)$, if any, define the bound states of the problem. Namely if $\lambda \in \mathbb{R}$ is such that

$$
U\left(s+\frac{2 \pi}{\omega}, s ; \mu\right) f=e^{-\frac{\lambda 2 \pi i}{\omega}} f, \quad f \in L^{2}\left(\mathbb{R}^{3}\right),
$$

then $U(t, s ; \mu) f=e^{-i(t-s) \lambda} f(t)$ with $f$ periodic in $t$ and localized in space;

(2) Let $\mathscr{K}$ be the Hilbert space $L^{2}\left(\mathbb{R}^{3}\right) \otimes L^{2}\left(T_{\omega}\right), T_{\omega}$ the circle $\mathbb{R} /(2 \pi / \omega) \mathbb{Z}$, and let $K(\mu)$ be the self-adjoint realization of $H(t, \mu)-i \frac{\partial}{\partial t}$ in $\mathscr{K}$. Then

$$
\sigma\left(U\left(s+\frac{2 \pi}{\omega}, s ; \mu\right)\right),
$$

the spectrum of $U(\cdot)$, is analyzed through $\sigma(K(\mu))$ in the following way: if $K(\mu) \phi(t)=\lambda \phi(t)$, then $\phi(t)$ is a $L^{2}$-valued, continuous and periodic function of $t$ with $U(t, 0 ; \mu) \phi(0)=e^{-i \lambda t} \phi(t)$, and conversely if

$$
U\left(\frac{2 \pi}{\omega}, 0 ; \mu\right) \phi_{0}=e^{-i \lambda 2 \pi / \omega} \phi_{0}
$$

then $\phi(t)=e^{i \lambda t} U(t, 0 ; \mu) \phi_{0}$ is such that $K(\mu) \phi(t)=\lambda \phi(t)$.

(3) Consider $K(0)=-i \frac{\partial}{\partial t}+H=-i \frac{\partial}{\partial t}-\Delta+V$. Then $\sigma(K(0))=\bigcup_{n=-\infty}^{+\infty}\{n \omega+\sigma(H)\}$, so that all eigenvalues of $H$ appear as embedded eigenvalues in $\sigma(K(0))$. For $\mu \neq 0$ small enough these eigenvalues turn into resonances of $K(\mu)$, and hence of (1.3), in the standard sense of dilation analyticity. More precisely: if

$$
K(\theta, \mu)=-i \frac{\partial}{\partial t}+H(t, \theta, \mu)=-i \frac{\partial}{\partial t}-e^{-2 \theta} \Delta+V\left(e^{\theta} x_{1}+\mu \omega^{-2} \cos \omega t, e^{\theta} x_{2}, e^{\theta} x_{3}\right),
$$

then $K(\theta, \mu)$ is a holomorphic family of operators in $\mathscr{K}$ for $|\operatorname{Im} \theta|<\frac{\pi}{2} ; \sigma_{\text {ess }}(K(\theta, \mu))$ $=\bigcup_{n=-\infty}^{+\infty}\left\{n \omega+e^{-2 \theta} \mathbb{R}_{+}\right\}$; for $\operatorname{Im} \theta \neq 0, K(\theta, 0)$ admits all points of $\sigma_{P}(K(0))$ as isolated eigenvalues, and any such eigenvalue is stable for $\mu>0$ small. The eigenvalues of $K(\theta, \mu)$ are independent of $\theta$, and for almost every $\omega$ have strictly negative imaginary part if $\operatorname{Im} \theta<0$.

(4) Let $\lambda_{1}, \lambda_{2}$ be isolated, simple eigenvalues of $H=-\Delta+V, \lambda_{1}-\lambda_{2}= \pm \omega$, with eigenvectors $\phi_{1}, \phi_{2}$. Then the solution $U(t, \theta ; \mu) \phi_{1}$ of $H(t, \theta, \mu) \psi=i \frac{\partial \psi}{\partial t}$ with 
initial state $\phi_{1}$ oscillates in time between the two states $\phi_{1}, \phi_{2}$ whose energy difference is $\pm \omega$. This "classical resonance" phenomenon displays the "photon" property of the electric field.

If now $V=\left(x_{1}^{2}+x_{2}^{2}+x_{3}^{2}\right)^{-1 / 2} \equiv\left(x_{1}^{2}+x_{\perp}^{2}\right)^{-1 / 2}$, we see that

$$
V\left(e^{\theta} x_{1}+\mu \omega^{-2} \cos \omega t, e^{\theta} x_{\perp}\right), \quad \theta=i \phi,
$$

is singular for $x_{1}=-\left(\omega^{-2} \mu \cos \omega t\right) \cos \phi,\left|x_{\perp}\right|=\left|\left(\omega^{-2} \mu \cos \omega t\right) \sin \phi\right|$. This represents a circle of square-root branch points. Therefore the critical assumption that $V\left(e^{\theta} x_{1}+\omega^{-2} \cos \omega t, e^{\theta} x_{\perp}\right)$ extends for all $t$ to a holomorphic family of compact operators from $H^{2}\left(\mathbb{R}^{3}\right)$ to $L^{2}\left(\mathbb{R}^{3}\right)$ in some strip $|\operatorname{Im} \theta|<\alpha, \alpha>0$, is not fulfilled by the Coulomb potential. We will sidestep this difficulty by means of a method, the exterior complex scaling, introduced by Simon [9] to overcome the very same problem arising in the attempt of defining molecular resonances in the BornOppenheimer approximation. Motivated by the fact that the above singularities are confined within a bounded sphere, the exterior scaling transformation on a function $f \in L^{2}\left(\mathbb{R}^{3}\right)$ replaces the unitary dilation $(S(\theta) f)(x)=e^{3 \theta / 2} f\left(e^{\theta} x\right), \theta \in \mathbb{R}$, by the following unitary map:

$$
(\hat{S}(\theta, R) f)(x)=[\operatorname{det} \partial S(\theta, R) x / \partial x]^{1 / 2} f(S(\theta, R) x),
$$

where $R>0$ is fixed, $\hat{x}=x /|x|$, and

$$
S(\theta, R) x=\left\{\begin{array}{lll}
x & \text { for }|x| \leqq R \\
\left(R+e^{\theta}(|x|-R)\right) \hat{x} & \text { for }|x| \geqq R .
\end{array}\right.
$$

Therefore the following conditions on the potential $V(x)$ will be assumed: (We always take $|\mu| \leqq 1$ and $|\omega| \geqq \Gamma^{-1}$ for some $\Gamma>0$.)

A1. There are $R>0, a>0$, such that the function $\theta \rightarrow V(S(\theta, R) x+\mu E), E=(1,0,0)$, is a $C_{0}(|x| \geqq R)$-valued holomorphic function of $\theta$ in $\mathbb{C}_{a}=\{\theta \in \mathbb{C}:|\operatorname{Im} \theta|<a\}$ for any fixed $\mu$, and a continuous function of $\mu$ for any fixed $\theta$.

Here $C_{0}(|x| \geqq R)$ is the set of all continuous functions from $\{x:|x| \geqq R\}$ to $\mathbb{C}$, vanishing as $|x| \rightarrow \infty$. (Note that A1 implies

$$
\sup _{t}\left|V\left(S(\theta, R) x+\mu \omega^{-2} \cos \omega t E\right)\right| \in L^{\infty}(|x| \geqq R)
$$

and $\lim _{|x| \rightarrow \infty} \sup _{t}\left|V\left(S(\theta, R) x+\mu \omega^{-2} \cos \omega t E\right)\right|=0$ uniformly on compacts with respect to $(\mu, \theta)$.)

A2. Let $\chi(R)$ be the characteristic function of the ball $|x| \leqq R$. Then:

$$
\sup _{|\varrho| \leqq \Gamma^{2}}\left|\chi(R) V\left(x_{1}+\varrho, x_{\perp}\right)\right|^{1 / 2} \in L^{q}, \quad 3<q \leqq \infty .
$$

A3. There exists a function $B(x)>0$ such that the following conditions are satisfied:

(i) For some $3<q \leqq \infty, B(x) \in L^{q}(|x| \leqq R)$ and $B \in C_{0}(|x| \geqq R)$.

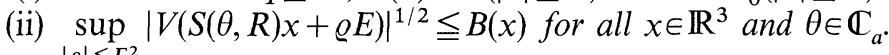
$|\varrho| \leqq \Gamma^{2}$ 
(iii) $B(x)^{-1} V\left(S(\theta, R) x+\mu \omega^{-2} \cos \omega t E\right)^{1 / 2}$ is a $C\left(T_{\omega}\right) \otimes C_{0}(|x| \geqq R)$-valued analytic function of $\theta \in \mathbb{C}_{a}$.

(iv) $\lim _{\mu \rightarrow 0}\left\|\left|V\left(S(\theta, R) x+\mu \omega^{-2} \cos \omega t E\right)^{1 / 2}-V(S(\theta, R) x)^{1 / 2}\right| B(x)^{-1}\right\|_{L^{\infty}\left(\mathbb{R}^{3} \times \mathbb{R}\right)}=0$.

A4. The function $t \rightarrow(-\Delta+1)^{-1 / 2} V\left(x+\mu \omega^{-2} \cos \omega t E\right)(-\Delta+1)^{-1 / 2}$, taking values in $B\left(L^{2}\right)$ by $A 1$ and $A 2$ above, is strongly differentiable in $t>0$.

It is easy to check that the Coulomb potential fulfills the above assumptions.

In the subsequent sections we will explicitly realize the holomorphic families associated with $K(0)$ and $K(\mu)$ through the analytic continuation of their unitary image under the exterior scaling. This cannot be obtained through a straightforward generalization of the arguments of [12] essentially because $V$ is too singular and the operator family $-S(\theta, R) \Delta S(\theta, R)^{-1}$, the exterior complex-scaled Laplace operator, is not normal for $\operatorname{Im} \theta \neq 0$. The main technique will consist in working out Kato's type estimates [2] with $-\Delta$ replaced by $-S(\theta, R) \Delta S(\theta, R)^{-1}$, so that the arguments of [13] can be generalized to this particular non-selfadjoint case.

In this way we will be able to extend to the present situation, beyond (2) and (3) above, the existence result for resonances. Furthermore we will see in Sect. 5 that under an additional condition described there which is also satisfied by the Coulomb potentials the imaginary part of the resonance is given by the Fermi Golden Rule to second order perturbation theory. In particular this implies that the width of such resonances does not vanish for almost all $\omega$ and sufficiently small $\mu$.

However, we were not able to prove in the present context the validity of the "photon property" recalled in (4) above. Here the main difficulty comes from the lack of an existence theorem for the time-dependent complex-scaled Schroedinger equations with potentials as singular as the Coulomb potential.

The reader is referred to [12] for all notations not explicitly recalled in this paper.

\section{Exterior Complex Scaling}

We collect in this section some results on the theory of exterior complex scaling introduced by Simon [9], strictly necessary in what follows. The reader is referred to $[10]$ for a complete treatment.

Let us denote by $x=\left(x_{1}, x_{2}, x_{3}\right)$, the generic point in $\mathbb{R}^{3} ; r=|x|$ $=\left(x_{1}^{2}+x_{2}^{2}+x_{3}^{2}\right)^{1 / 2}$ is its length, and $\hat{x}=x /|x|$ its angular vector. For $R \geqq 0$ we define a one-parameter group of exterior scaling transformations $S(\theta, R), \theta \in \mathbb{R}$, by

$$
S(\theta, R) x=\left\{\begin{array}{lll}
x & \text { for } & |x| \leqq R \\
\left(R+e^{\theta}(|x|-R)\right) \hat{x} & \text { for } & |x| \geqq R
\end{array}\right.
$$

This group has a natural unitary representation $\hat{S}(\theta, R)$ on $\mathscr{H}=L^{2}\left(\mathbb{R}^{3}\right)$, given by

$$
(\hat{S}(\theta, R) f)(x)=[\operatorname{det} \partial S(\theta, R) x / \partial x]^{1 / 2} f(S(\theta, R) x),
$$

where, as is easily checked

$$
[\operatorname{det} \partial S(\theta, R) x) / \partial x]^{1 / 2}=\left\{\begin{array}{lll}
1 & \text { for } & |x| \leqq R \\
e^{\theta / 2}\left(R+e^{\theta}(|x|-R)\right) /|x| & \text { for } & |x|>R
\end{array}\right.
$$


Let $H_{0}$ be the unique self-adjoint extension of $-\Delta$ defined on $C_{0}^{\infty}\left(\mathbb{R}^{3}\right)$. We define the exterior scaled free Hamiltonian $H_{0}$ in the following way:

$$
H_{0}(\theta, R)=\hat{S}(\theta, R) H_{0} \hat{S}(\theta, R)^{-1}, \quad \theta \in \mathbb{R} .
$$

2.1. Proposition. Let $\theta \in \mathbb{R}$ and $R>0$. Then

$$
\begin{aligned}
D\left(H_{0}(\theta, R)\right)= & \left\{f: f=f_{-} \oplus f_{+} \in H^{2}(|x|<R) \oplus H^{2}(|x|>R),\right. \\
& \text { and } \lim _{r \uparrow R} f_{-}(r \hat{x})=e^{-\theta / 2} \lim _{r \downarrow R} f_{+}(r \hat{x}), \\
& \left.\lim _{r \uparrow R} \frac{\partial}{\partial r}\left(r f_{-}(r \hat{x})\right)=e^{-3 \theta / 2} \lim _{r \downarrow R} \frac{\partial}{\partial r}\left(r f_{+}(r \hat{x})\right)\right\},
\end{aligned}
$$

and for $f \in D\left(H_{0}(\theta, R)\right)$

$$
\begin{aligned}
H_{0}(\theta, R) f(x)= & -\Delta f_{-}(x) \oplus\left(-e^{-2 \theta} \Delta f_{+}(x)-\frac{x^{2}-(S(\theta, R) x)^{2} e^{-2 \theta}}{(S(\theta, R) x)^{2}}\right. \\
& \left.\cdot\left(\Delta-\frac{2}{|x|} \frac{\partial}{\partial|x|}-\sum_{j, k=1}^{3} \hat{x}_{j} \hat{x}_{k} \frac{\partial^{2}}{\partial x_{j} \partial x_{k}}\right) f_{+}(x)\right) .
\end{aligned}
$$

Here the limits are taken in $L^{2}\left(S^{2}\right), S^{2}$ the unit sphere in $\mathbb{R}^{3}$.

To prove Proposition 2.1 and for later convenience we introduced a unitary transformation $\mathcal{O}$ from $L^{2}\left(\mathbb{R}^{3}\right)$ to $L^{2}\left(\mathbb{R}_{+}, L^{2}\left(S^{2}\right), d r\right)$, defined by

$$
\mathcal{O} f(r, \omega)=r f(r \omega), \quad r \geqq 0, \quad \omega \in S^{2} .
$$

As is well known, $\mathcal{O}$ is the transformation into polar coordinates and

$$
\mathcal{O} H_{0} \mathcal{O}^{-1}=-\frac{d^{2}}{d r^{2}}+\frac{\Lambda}{r^{2}}
$$

where $\Lambda$ is the Laplace-Beltrami operator in $S^{2}$. By a simple computation we see that

$$
\mathcal{O}\left(\hat{S}(\theta, R) \mathcal{O}^{-1} f(r, \omega)= \begin{cases}f(r, \omega), & 0 \leqq r<R \\ e^{\theta / 2} f\left(R+e^{\theta}(r-R), \omega\right), & R>r\end{cases}\right.
$$

and

$$
\mathcal{O} H_{0}(\theta, R) \mathcal{O}^{-1} f(r, \omega)= \begin{cases}-\frac{d^{2} f}{d r^{2}}+\frac{\Lambda f}{r^{2}}, & 0 \leqq r<R, \\ -e^{-2 \theta} \frac{d^{2} f}{d r^{2}}+\frac{\Lambda f}{\left(R+e^{\theta}(r-R)\right)^{2}}, & R>r .\end{cases}
$$

Proposition 2.1 can be proved by using (2.8)-(2.10) and the change of variables. We omit the details.

Hereafter $R>0$ is fixed and we shall often omit the dependence on $R$ in the following expressions. We assume $|a|<\pi / 4$ in what follows.

Now we wish to extend the variable $\theta$ in the complex plane in such a way that $H_{0}(\theta, R)$ represents a holomorphic family of operators in $\theta$. For 
$\theta \in \mathbb{C}_{a}=\{\theta:|\operatorname{Im} \theta|<a\}$, we define a quadratic form $Q(\theta)$ as :

$$
D(Q(\theta))=\left\{f \in H^{1}(|x|<R) \oplus H^{1}(|x|>R): \lim _{r \uparrow R} f_{-}(r \hat{x})=e^{-\theta / 2} \lim _{r \downarrow R} f_{+}(r \hat{x})\right\},
$$

$$
\begin{aligned}
& Q(\theta)(f, g)=\int_{|x| \leqq R} \nabla f \cdot \overline{\nabla g} d x \\
& \quad+\int_{R}^{\infty} d r \cdot \int_{S^{2}}\left\{e^{-2 \theta} \frac{d}{d r} \mathcal{O} f \cdot \frac{d}{d r} \overline{\mathcal{O} g}+\left(R+e^{\theta}(r-R)\right)^{-2} \sqrt{\Lambda} \mathcal{O} f(r, \omega) \cdot \sqrt{\Lambda} \overline{\mathcal{O} g(r, \omega)}\right\} d \omega .
\end{aligned}
$$

To simplify the notation, we write $\Omega_{-}=\{|x|<R\}, \Omega_{+}=\{|x|>R\}$, and

$$
J(x, \theta)=(\operatorname{det} \partial S(\theta, x) / \partial x)^{1 / 2},
$$

where the positive branch is taken for $\theta \in \mathbb{R}$.

2.2. Proposition. (1) If $\theta \in \mathbb{R}, Q(\theta)$ is the quadratic form associated with the selfadjoint operator $H_{0}(\theta, R)$.

(2) For $\theta \in \overline{\mathbb{C}}_{a}^{ \pm}, Q(\theta)$ is a closed, strictly sectorial form with numerical range:

$$
W_{0}(\theta)=\{z: 0 \leqq \mp \arg z \leqq \mp 2 \operatorname{Im} \theta\} .
$$

Proof. We first show (2). Let $\left\{f_{n}\right\}$ be a sequence in $D(Q(\theta))$ such that $f_{n} \rightarrow f$ in $\mathscr{H}$ and $Q(\theta)\left(f_{n}-f_{m}, f_{n}-f_{m}\right) \rightarrow 0$, as $n, m \rightarrow \infty$. Writing $\theta=\xi+i \eta, e^{\zeta}(r-R)=\tau$, we see that for $|\eta|<a$

$$
\operatorname{Re}\left(R+e^{i \eta} \tau\right)^{-2}=\frac{R^{2}+2 R \tau \cos \eta+\tau^{2} \cos 2 \eta}{\left(R^{2}+2 \cos \eta R \tau+\tau^{2}\right)^{2}} \geqq \frac{\cos 2 a}{(R+\tau)^{2}} .
$$

Thus by taking the real part of $Q(\theta)\left(f_{n}-f_{m}, f_{n}-f_{m}\right)$, we obtain

$$
\left\|\nabla\left(f_{n}-f_{m}\right)\right\|_{L^{2}\left(\Omega_{-}\right)} \rightarrow 0, \quad\left\|d \mathcal{O} f_{m, n}(r, \omega) / d r\right\|_{L^{2}\left([R, \infty), L^{2}\left(S^{2}\right), d r\right)} \rightarrow 0,
$$

and

$$
\left\|r^{-1} \sqrt{\Lambda} \mathcal{O} f_{m, n}(r, \omega)\right\|_{L^{2}\left([R, \infty), L^{2}\left(S^{2}\right), d r\right)} \rightarrow 0, \quad f_{m, n}=f_{m}-f_{n} .
$$

From this we conclude that $f_{n} \rightarrow f$ both in $H^{1}(|x|<R)$ and $H^{1}(|x|>R)$. Since the trace operators on the boundaries $|x|=R$ are continuous both from $H^{1}(|x|<R)$ and $H^{1}(|x|>R)$ to $L^{2}(|x|=R), f$ satisfies the boundary condition and we have the closedness of $Q(\theta)$.

Since

$$
0 \leqq-\operatorname{Im}\left(R+e^{i \eta} \tau\right)^{2}=\frac{2 \tau \sin \eta(R+\tau \cos \eta)}{\left(R^{2}+2 \cos \eta R \tau+\tau^{2}\right)^{2}} \leqq \tan 2 \eta \operatorname{Re}\left(R+e^{i \eta} \tau\right)^{2},
$$

it is easy to see that the numerical range is given by (2.13).

To show (1) it is now sufficient to prove that, for $f, g \in D\left(H_{0}(\theta)\right),\left\langle H_{0}(\theta) f, g\right\rangle$ $=Q(\theta)(f, g)$. Since this represents a simple computation, integrating by parts and using the boundary condition together with (2.10) and (2.12), we omit the details. 
Now by Proposition 2.2 and a general theorem (cf. Kato [2], Theorem VI.2.1) there exists a unique $m$-sectorial operator $H_{0}(\theta, R)$ such that $Q(\theta)$ is its associated quadratic form for any $\theta \in \mathbb{C}_{a}^{ \pm}\left(0 \leqq a<\frac{\pi}{4}\right)$.

2.3. Proposition. For $\theta \in \mathbb{C}_{a}^{ \pm}$and $R>0$, the domain and the action of the operator $H_{0}(\theta, R)$ are given by (2.5) and (2.6), respectively.

Proof. Let us denote by $\dot{\mathscr{D}}(\theta)$ the right hand side of $(2.5)$, and by $T_{0}(\theta)$ the formal operator in the right hand side of (2.6). Clearly $\dot{\mathscr{D}}(\theta) \subset D(Q(\theta))$, and by (2.10) after integrating by parts, we get

$$
\left\langle T_{0}(\theta) f, g\right\rangle=Q(\theta)(f, g), \quad f \in \dot{\mathscr{D}}(\theta), \quad g \in D\left(Q_{0}(\theta)\right) .
$$

Thus $T_{0}(\theta)$ with domain $\dot{\mathscr{D}}(\theta)$ is a restriction of $H_{0}(\theta, R)$ (cf. ibidem Corollary VI.2.4). Let us show that $\dot{\mathscr{D}}(\theta) \supset D\left(H_{0}(\theta, R)\right)$. By its construction (cf. ibidem p. 324). $f \in D\left(H_{0}(\theta, R)\right)$, if and only if there is $u \in \mathscr{H}$ such that $Q(\theta)(f, g)=\langle u, g\rangle$ for all $g \in D(Q(\theta))$.

Taking $g \in C_{0}^{\infty}\left(\mathbb{R}^{3} \backslash \partial \Omega\right) \subset D(Q(\theta))$, we see that $T_{0}(\theta) f=u$ in $\mathscr{D}^{\prime}\left(\Omega_{-} \cup \Omega_{+}\right)$. Since $T_{0}(\theta)=-\Delta$ for $|x|<R$, and, for $|x|>R, T_{0}(\theta)$ is uniformly strongly elliptic with coefficients converging to constants such that $T_{0}(\theta) \sim-e^{-2 \theta} \Delta$ at infinity, by the elliptic regularity theorem near the boundary and at infinity (see e.g. Mizohata [6], pp. 213 and 222), $f \in H^{2}(|x|>R) \oplus H^{2}(|x|<R)$. Now using (2.10) once more with $g \in C^{\infty}(|x| \leqq R) \cap C^{\infty}(|x| \geqq R) \cap D(Q(\theta))$, and performing a partial integration we see that $f$ has to satisfy the additional boundary condition $\lim _{r \uparrow R} \frac{\partial}{\partial r}(r f(r \hat{x}))$ $=\lim _{r \downarrow R} e^{-3 \theta / 2} \frac{\partial}{\partial r}(r f(r \hat{x}))$.

We write $J(\theta, x)=e^{\theta / 2}\left(R+e^{\theta}(|x|-R)\right) /|x|$ for $\theta \in \mathbb{C}_{a}$. Let $\chi_{ \pm}(x)$ denote the characteristic function of $\Omega_{ \pm}$. For $\theta \in \mathbb{C}_{a}$ and $z \in \mathbb{C}$ we set:

$$
\begin{aligned}
& G_{\theta}^{--}(z, \dot{x}, y)=\frac{1}{4 \pi} \frac{\exp (-\sqrt{-z}|x-y|)}{|x-y|} \chi_{-}(x) \chi_{-}(y), \\
& G_{\theta}^{-+}(z, x, y)=\frac{1}{4 \pi} \frac{\exp \left(-\sqrt{-z}\left\{(x-S(\theta) y)^{2}\right\}^{1 / 2}\right)}{\left\{(x-S(\theta) y)^{2}\right\}^{1 / 2}} \chi_{-}(x) \chi_{+}(y) J(\theta, y), \\
& G_{\theta}^{+-}(z, x, y)=\frac{1}{4 \pi} \frac{\exp \left(-\sqrt{-z}\left\{(S(\theta) x-y)^{2}\right\}^{1 / 2}\right)}{\left\{(S(\theta) x-y)^{2}\right\}^{1 / 2}} \chi_{+}(x) \chi_{-}(y) J(\theta, x), \\
& G_{\theta}^{++}(z, x, y)=\frac{1}{4 \pi} \frac{\exp \left(-\sqrt{-z}\left\{(S(\theta) x-S(\theta) y)^{2}\right\}^{1 / 2}\right)}{\left\{(S(\theta) x-S(\theta) y)^{2}\right\}^{1 / 2}} \chi_{+}(x) \chi_{+}(y) J(\theta, x) J(\theta, y) .
\end{aligned}
$$

Correspondingly we define the operators

$$
G_{\theta}^{--}(z) f(x)=\int_{\mathbb{R}^{3}} G_{\theta}^{--}(z, x, y) f(y) d y, \text { etc. },
$$


and

$$
G_{\theta}(z)=G_{\theta}^{--}(z)+G_{\theta}^{-+}(z)+G_{\theta}^{+-}(z)+G_{\theta}^{++}(z) .
$$

Here in the right hand side of (2.15), we take $\sqrt{-z} \geqq 0$ for $z \leqq 0$, and the positive branch of $\{\cdot\}^{1 / 2}$ for $\theta \in \mathbb{R}$ (unless $x=y$ ).

Note that

$$
\begin{array}{lll}
G_{\theta}^{-+}(z, x, y) \sim e^{\theta / 2} \frac{\exp \left(-\sqrt{-z} e^{\theta}|y|\right)}{4 \pi|y|} \quad \text { as } & |y| \rightarrow \infty, \\
G_{\theta}^{+-}(z, x, y) \sim e^{\theta / 2} \frac{\exp \left(-\sqrt{-z} e^{\theta}(|x|)\right.}{4 \pi|x|} \quad \text { as } & |x| \rightarrow \infty, \\
G_{\theta}^{++}(z, x, y) \sim e^{2 \theta} \frac{\exp \left(-\sqrt{-z} e^{\theta}|x-y|\right)}{4 \pi|x-y|} \quad \text { as } \begin{array}{l}
|x|,|y| \rightarrow \infty, \\
|x-y| \rightarrow \infty .
\end{array}
\end{array}
$$

2.4. Proposition. Let $\theta \in \mathbb{C}_{a}$ and $G_{\theta}(z)$ be defined by (2.15)-(2.17). Then for any $\theta \in \mathbb{C}_{a}, G_{\theta}(z)$ is a bounded-operator valued analytic function of $z$ in $\{z:-2 \operatorname{Im} \theta$ $<\arg z<2 \pi-2 \operatorname{Im} \theta\}$ on the Riemann surface of $\sqrt{-z}$; for any $z$ in the first Riemann sheet of $\sqrt{-z}$ it is a bounded-operator valued analytic function of $\theta$ in the domain $\left\{\theta \in \mathbb{C}_{a}:-\frac{1}{2} \arg z<\operatorname{Im} \theta<\pi-\frac{1}{2} \arg z\right\}$.

Proof. Except for the analyticity in $\theta$, the statements are almost obvious. However for later convenience let us prove somewhat more detailed estimates than those stated above when $z$ varies along lines parallel to the real axis. We first show the boundedness and analyticity (in $z$ ) for any fixed $\theta \in \mathbb{C}_{a}^{+}$. The case $\theta \in \mathbb{C}_{a}^{-}$can be treated in the same way, and the case $\theta \in \mathbb{R}$ is easy.

(i) $G_{\theta}^{--}(z)$ is $\theta$-independent. When $z$ ranges over the sector $\{z:-2 \operatorname{Im} \theta<\arg z$ $<2 \pi-2 \operatorname{Im} \theta\}$ of the Riemann surface of $\sqrt{-z}$, along a line $z=a+i b, b$ fixed, we have

$$
\begin{gathered}
\operatorname{Re}-\sqrt{-z} \leqq 0 \text { if } b \geqq 0, \\
\operatorname{Re}-\sqrt{-z} \leqq C_{a} \text { if } b \leqq 0, \text { for some } C_{a}>0 .
\end{gathered}
$$

Thus there is $M_{a}>0$ such that, along this line:

$$
\left\|G_{\theta}^{--}(z)\right\|^{2} \leqq\left\|G_{\theta}^{--}(z)\right\|_{\text {H.S. }}^{2} \leqq(4 \pi)^{-2} \iint_{\Omega_{-}} \frac{\exp \left(2 C_{a}|x-y|\right)}{|x-y|^{2}} d x d y<M_{a} .
$$

The analyticity in $z$ is obvious.

(ii) Write now $\theta=\xi+i \eta, \eta>0, \sigma=x-R \hat{y}$,

$$
\tau=e^{\xi}(|y|-R) \hat{y} \quad \text { for } \quad x \in \Omega_{-}, y \in \Omega_{+} .
$$

By the unitary equivalence, it is enough to consider the case $\xi=0$. Clearly

$$
\begin{gathered}
\langle\sigma, \tau\rangle \leqq 0, \\
(x-S(\theta) y)^{2}=\left(\sigma-e^{i \eta} \tau\right)^{2}=\sigma^{2}-2 \sigma \tau \cos \eta+\tau^{2} \cos 2 \eta+i\left(\tau^{2} \sin 2 \eta-2 \sigma \tau \sin \eta\right) .
\end{gathered}
$$


From (2.23) it is easy to see that

$$
0 \leqq \arg \left\{(x-S(\theta) y)^{2}\right\}^{1 / 2} \leqq \eta,
$$

and that, for any $\varepsilon>0$, there is $M_{\varepsilon}>0$ such that, for $|y| \geqq M_{\varepsilon}$

$$
\begin{gathered}
0 \leqq \eta-\varepsilon \leqq \arg \left\{(x-S(\theta) y)^{2}\right\}^{1 / 2} \leqq \eta \quad(\eta>0), \\
\cos 2 \eta(x-R \hat{y}-(|y|-R) \hat{y})^{2} \leqq\left|(x-S(\theta) y)^{2}\right| \leqq(x-R \hat{y}-(|y|-R) \hat{y})^{2} .
\end{gathered}
$$

Hence we have the estimates:

$$
\begin{gathered}
\operatorname{Re}-\sqrt{-z}\left\{(x-S(\theta) y)^{2}\right\}^{1 / 2} \leqq C_{\varepsilon, b} \quad \text { on } \quad z=a+i b,|y| \leqq M_{\varepsilon}, \\
\operatorname{Re}-\sqrt{-z}\left\{(x-S(\theta) y)^{2}\right\}^{1 / 2} \leqq-\cos 2 \eta \sin (\eta-\varepsilon)|x-R \hat{y}-(|y|-R) \hat{y}||z|^{1 / 2} \\
\text { on } \quad z=a+i b, b \geqq 0,|y| \geqq M_{\varepsilon} .
\end{gathered}
$$

If $z=a+i b, b<0$, and $z$ ranges only over a closed half-line not intersecting the halfline $e^{-2 \theta} \mathbb{R}_{+}$, so that $\frac{\pi}{2}+2 \varepsilon-\eta<\arg (-\sqrt{-z})<\frac{3}{2} \pi-2 \varepsilon-\eta$, an estimate analogous to (2.28) holds with $\sin \varepsilon$ in place of $\sin (\eta-\varepsilon)$ in the right hand side. Combining these estimates, we have for $z=a+i b, a \in \mathbb{R}, b<0, a$ ranging over half-lines of $\mathbb{R}$ such that $z$ does not intersect $e^{-2 \theta} \mathbb{R}_{+}$:

$$
\begin{gathered}
\left\|G_{\theta}^{-+}(z)\right\|^{2} \leqq\left\|G_{\theta}^{-+}(z)\right\|_{\text {H.S. }}^{2} \leqq \int_{|x| \leqq R} d x \int_{R \leqq|y| \leqq M_{\varepsilon}} C_{\varepsilon, \theta, R}(\sqrt{\cos 2 \eta}|x-R \hat{y}-(|y|-R) \hat{y}|)^{-2} \\
\quad+C_{\theta} \int_{|x| \leqq R} d x \int_{|y| \geqq M_{\varepsilon}} d y \frac{\exp \left(-C_{\varepsilon, \eta}|z|^{1 / 2}|x-R \hat{y}-(|y|-R) \hat{y}|\right)}{\cos 2 \eta|\mathrm{x}-R \hat{\mathrm{y}}-(|\mathrm{y}|-R) \hat{\mathrm{y}}|^{2}} \leqq C_{R, \theta}<\infty .
\end{gathered}
$$

(iii) For $G_{\theta}^{+-}(z)$, we can apply the above estimates on the integral kernel, interchanging the roles of $x$ and $y$, to get an analogous uniform bound. We omit the details.

(iv) Let us now consider $G_{\theta}^{++}(z)$. By unitary equivalence, it is enough to consider the case $\theta=i \eta, \eta \geqq 0$. We have:

$$
\begin{aligned}
(S(\theta) x-S(\theta) y)^{2}= & e^{2 \theta}(x-y)^{2}+2 e^{\theta}\left(1-e^{\theta}\right) R(x-y) \cdot(\hat{x}-\hat{y}) \\
& +\left(1-e^{\theta}\right)^{2} R^{2}(\hat{x}-\hat{y})^{2}=\left\{\cos 2 \eta(x-y-R \hat{x}+R \hat{y})^{2}\right. \\
& \left.+2 \cos \eta(|x|+|y|-2 R)(1-\hat{x} \cdot \hat{y})+R^{2}(\hat{x}-\hat{y})^{2}\right\} \\
& +i\left\{\sin 2 \eta(x-y-R \hat{x}+R \hat{y})^{2}+2 \sin \eta(|x|+|y|-2 R)(1-\hat{x} \cdot \hat{y})\right\} .(2
\end{aligned}
$$

Using the identity

$$
(\hat{x}-\hat{y}) \cdot(x-y-R(\hat{x}-\hat{y}))=(|x|+|y|-2 R)(1-\hat{x} \cdot \hat{y}),
$$

together with (2.29), we get:

$$
\begin{gathered}
\cos 2 \eta|x-y|^{2} \leqq|S(\theta) x-S(\theta) y|^{2} \leqq|x-y|^{2}, \\
0 \leqq \arg \left((S(\theta) x-S(\theta) y)^{2}\right)^{1 / 2} \leqq \eta .
\end{gathered}
$$

Moreover for any $\varepsilon>0$, there is $M_{\varepsilon}>0$ such that for $|x-y|>M_{\varepsilon}$

$$
\eta-\varepsilon \leqq \arg \left((S(\theta) x-S(\theta) y)^{2}\right)^{1 / 2} \leqq \eta .
$$


By means of (2.31)-(2.33) we can see that

$$
\begin{aligned}
& \sup _{x} \int_{|y|>R}\left|G_{\theta}^{++}(z, x, y)\right| d x \leqq \sup _{x} \int_{|x-y| \leqq M_{\varepsilon}} C_{\varepsilon}(\sqrt{\cos 2 \eta}|x-y|)^{-1} d y \\
& \quad+\sup _{x} \int_{|x-y| \geqq M_{\varepsilon}} C_{\theta}^{\prime \prime}(\sqrt{\cos 2 \eta}|x-y|)^{-1} \exp \left(-C_{\theta}^{\prime}|z|^{1 / 2}|x-y|\right) d y \leqq D_{\theta, \varepsilon}<\infty,
\end{aligned}
$$

whenever $z$ ranges over a line $z=a+i b, b \geqq 0$ or two half-lines $z=a+i b, b<0$ taken as in the case (ii). A similar estimate holds for $\sup _{y} \int\left|G_{\theta}^{++}(z, x, y)\right| d x$, and this proves the uniform boundedness.

(v) Finally let us prove the analyticity in $\theta$.

The proof above shows that $\left\|G_{\theta}(z)\right\|$ is locally uniformly bounded in $\theta$. Then it suffices to show that $\left\langle G_{\theta}(z) f, g\right\rangle$ is analytic for $f, g \in C_{0}^{\infty}\left(\Omega_{+} \cup \Omega_{-}\right)$, since the latter space is a dense set in $\mathscr{H}$. We show the analyticity of $\left\langle G_{\theta}^{++}(z) f_{+}, g_{+}\right\rangle$, $\left\langle G_{\theta}^{+-}(z) f_{-}, g_{+}\right\rangle,\left\langle G_{\theta}^{-+}(z) f_{+}, g_{-}\right\rangle .\left\langle G_{\theta}^{--}(z) f_{-}, g_{-}\right\rangle$is trivially analytic. Here $\left(f_{+}, g_{+}\right) \in C_{0}^{\infty}\left(\Omega_{+}\right),\left(f_{-}, g_{-}\right) \in C_{0}^{\infty}\left(\Omega_{-}\right)$.

On the support of $f_{-}(y) g_{+}(x)$ [respectively $\left.f_{+}(y) g_{-}(x)\right] G_{\theta}^{+-}(z, x, y)$ [respectively $\left.G_{\theta}^{-+}(z, y, y)\right]$ is analytic in $\theta,(x, y)$ fixed. Using the estimates in (ii) $\left[\right.$ respectively (iii)] we see that $\left\langle G_{\theta}^{+-}(z) f_{-}, g_{+}\right\rangle\left[\right.$respectively $\left.\left\langle G_{\theta}^{-+}(z) f_{+}, g_{-}\right\rangle\right]$is analytic in $\theta$. To see the analyticity in $\theta$ of $\left\langle G_{\theta}^{++}(z) f_{+}, g_{+}\right\rangle$, let us approximate it by an operator $G_{\theta, n}^{++}$, whose kernel is given by $G_{\theta, n}^{++}(z, x, y)=G_{\theta}^{++}(z, x, y)$ $\chi\left(|x-y|>\frac{1}{n}\right)$. By the estimates (iv) it is clear that $\left\langle G_{\theta, n}^{++}(z) f_{+}, g_{+}\right\rangle$is analytic in $\theta$ and it converges to $\left\langle G_{\theta}^{++}(z) f_{+}, g_{+}\right\rangle$locally uniformly in $\theta$ as $n \rightarrow \infty$. This completes the proof.

2.5. Corollary. Let $\theta \in \mathbb{C}_{a}^{ \pm}$and $z \notin\left\{e^{-2 \theta} \mathbb{R}_{+}-\omega \mathbb{Z}\right\}=\left\{e^{-2 \theta} x-\omega k: x>0, \quad k \in \mathbb{Z}\right\}$. Then

$$
\sup _{n}\left\|G_{\theta}(z+n \omega)\right\|=C(\theta, z)<+\infty
$$

where $z+n \omega$ runs over the sector $W=\{w:-2 \operatorname{Im} \theta<\arg w<2 \pi-2 \operatorname{Im} \theta\}$ of the Riemann surface of $\sqrt{-z}$.

This corollary is proved in the proof of Proposition 2.4.

2.6. Proposition. Let $\theta \in \mathbb{C}_{a}$. Then

(1) $\sigma\left(H_{0}(\theta, R)\right)=\sigma_{\text {ess }}\left(H_{0}(\theta, R)\right)=e^{-2 \theta} \mathbb{R}_{+}$.

(2) If $z \notin \sigma\left(H_{0}(\theta, R)\right),\left(H_{0}(\theta, R)-z\right)^{-1}=G_{\theta}(z)$.

Proof. For $\theta \in \mathbb{R},(1)$ and (2) are easy to obtain from the definition and the well known formula $(-\Delta-z)^{-1} f(x)=(4 \pi)^{-1} \int|x-y|^{-1} \exp (-\sqrt{-z}|x-y|) f(y) d y$. Thus for $\left(z, z^{\prime}\right) \notin \mathbb{R}$, we have

$$
G_{\theta}(z)-G_{\theta}\left(z^{\prime}\right)=G_{\theta}(z) G_{\theta}\left(z^{\prime}\right)\left(z-z^{\prime}\right), \quad \theta \in \mathbb{R} .
$$

Since both sides of (2.35) are analytic in $\theta$ by Proposition $2.4,(2.35)$ holds for $\theta$ in $\left\{\theta:-\frac{1}{2} \arg z<\operatorname{Im} \theta<\pi-\frac{1}{2} \arg z\right\} \cap\left\{\theta:-\frac{1}{2} \arg z^{\prime}<\operatorname{Im} \theta<\pi-\frac{1}{2} \arg z^{\prime}\right\} \cap \mathbb{C}_{a} . \quad$ Then, using the same argument first for $z$ and then for $z^{\prime}$, we see that for any $\theta \in \mathbb{C}_{a},(2.35)$ 
holds for $z, z^{\prime} \in\{z:-2 \operatorname{Im} \theta<\arg z<2 \pi-2 \operatorname{Im} \theta\}$. By a standard computation we have that $G_{\theta}(z) f \in D\left(H_{0}(\theta, R)\right)$ if $f \in C_{0}^{\infty}\left(\mathbb{R}^{3}\right)$. Since, for $\theta \in \mathbb{R}, z \notin \mathbb{R}, f, g \in C_{0}^{\infty}\left(\mathbb{R}^{3}\right)$ :

$$
\left\langle G_{\theta}(z) f,\left(H_{0}(\theta, R)-z\right)^{*} g\right\rangle=\langle f, g\rangle,
$$

and the left hand side is an analytic function of $\theta$ (and $z$ ), we see that for any $f \in C_{0}^{\infty}\left(\mathbb{R}^{3}\right)$ and $-2 \operatorname{Im} \theta<\arg z<2 \pi-2 \operatorname{Im} \theta$ :

$$
\left(H_{0}(\theta, R)-z\right) G_{\theta}(z) f=f \quad \text { in } \quad \mathscr{D}^{\prime}\left(\mathbb{R}^{3}\right) .
$$

It follows then that (2.37) holds for all $f \in \mathscr{H}$, since $H_{0}(\theta, R)-z$ is a closed operator. This implies that $G_{\theta}(z)^{-1}$ exists and is the inverse of an operator which is of course $\left(H_{0}(\theta, R)-z\right)$. This proves the second statement and also that $\sigma\left(H_{0}(\theta, R)\right) \subset e^{-2 \theta} \mathbb{R}_{+}$. Now the converse statement, $e^{-2 \theta} \mathbb{R}_{+} \supset \sigma\left(H_{0}(\theta, R)\right)$, can be easily shown by using a sequence of trial functions, and this proves (1).

Let us now study some properties of the semigroup $\exp \left(\mp i t H_{0}(\theta, R)\right), \theta \in \overline{\mathbb{C}}_{a}^{ \pm}$, generated by $H_{0}(\theta, R)$, which will turn out to yield the key estimates needed in what follows.

2.7. Lemma. Let $\theta \in \overline{\mathbb{C}}_{a}^{ \pm}$. Then:

(1) $\mp i H_{0}(\theta, R)$ generates a strongly continuous contraction semi-group $\exp \left(\mp i t H_{0}(\theta, R)\right), t \geqq 0$, on the Hilbert space $\mathscr{H}=L^{2}\left(\mathbb{R}^{3}\right)$.

(2) For $\theta \in \overline{\mathbb{C}}_{a}^{ \pm}, t>0$, and $f \in L^{1} \cap L^{2}$, $\exp \left(-i t H_{0}(\theta, R)\right) f$ can be expressed as

$$
\exp \left(-i t H_{0}(\theta, R)\right) f=F_{\theta}^{--}(t) f+F_{\theta}^{-+}(t) f+F_{\theta}^{+-}(t) f+F_{\theta}^{++}(t) f,
$$

where $F_{\theta}^{--}(t)$, etc., are the integral operators

$$
F_{\theta}^{--}(t) f(x)=\int_{\mathbb{R}^{3}} F_{\theta}^{--}(t, x, y) f(y) d y, \quad \text { etc. },
$$

whose kernels are given by:

$$
\left\{\begin{array}{l}
F_{\theta}^{--}(t)=(4 \pi i t)^{-3 / 2} \exp \left(-\frac{|x-y|^{2}}{i t}\right) \chi_{-}(x) \chi_{-}(y), \\
F_{\theta}^{-+}(t)=(4 \pi i t)^{-3 / 2} \exp \left(i \frac{(x-S(\theta) y)^{2}}{t}\right) J(y, \theta) \chi_{-}(x) \chi_{+}(y), \\
F_{\theta}^{+-}(t)=(4 \pi i t)^{-3 / 2} \exp \left(i \frac{(S(\theta) x-y)^{2}}{t}\right) J(x, \theta) \chi_{+}(x) \chi_{-}(y), \\
F_{\theta}^{++}(t)=(4 \pi i t)^{-3 / 2} \exp \left(i \frac{(S(\theta) x-S(\theta) y)^{2}}{t}\right) J(x, \theta) J(y, \theta) \chi_{+}(x) \chi_{+}(y) .
\end{array}\right.
$$

Similar statements hold for $t<0$ and $\theta \in \mathbb{C}_{a}^{-}$.

(3) There is a constant $C>0$ independent of $\theta \in \overline{\mathbb{C}}_{a}^{ \pm}, t>0$, and $f \in L^{1} \cap L^{2}$, such that

$$
\| \exp \left(\mp i t H_{0}(\theta, R) f\left\|_{\infty} \leqq C|t|^{-3 / 2}\right\| f \|_{1} .\right.
$$

Proof. (1) Since $H_{0}(\theta, R)$ is strictly $m$-sectorial with numerical range (2.13), the first statement is a well known result from the Hille-Yosida theory of semi-groups. 
(2) The second statement can be obtained from the Laplace inversion formula from the corresponding formulae (2.15)-(2.17) for the resolvent and Proposition 2.6(2). Since this computation is elementary (see e.g. Kato [1, IX.1.8]), we omit the details here.

(3) As we have computed in the proof of Proposition 2.4 [cf. (2.23) and (2.29)], the exponents have non-positive real parts. By unitary equivalence, it is enough to prove (2.41) for $\theta=i \eta$. In this case $|J(x, \theta)| \leqq 1$, so that

$$
\left\|\exp \left(\mp i t H_{0}(\theta, R)\right) f\right\|_{\infty} \leqq 4(4 \pi|t|)^{-3 / 2}\|f\|_{1} .
$$

2.8. Corollary. Let $f, g \in L^{p}\left(\mathbb{R}^{3}\right), 2 \leqq p \leqq \infty$, and denote by $F$ and $G$ the multiplication operators by $f$ and $g$ in $\mathscr{H}$. Then there is $C_{p}>0$ independent of $\theta \in \overline{\mathbb{C}}_{a}^{ \pm}, t>0$, such that

$$
\left\|F \exp \left(\mp i t H_{0}(\theta, R)\right) G\right\| \leqq C_{p}|t|^{-3 / p}\|f\|_{p}\|g\|_{p} .
$$

Moreover if $f$ and $g$ have compact support, $F \exp \left(\mp i t H_{0}(\theta, R)\right) G$ is norm continuous in $t>0$.

Proof. The first statement follows from (1), (3) of Lemma 2.7 and a well known interpolation argument (see Kato [2]). The second statement is obvious.

2.9. Corollary. Let $f, g \in L^{p}\left(\mathbb{R}^{3}\right) \cap L^{q}\left(\mathbb{R}^{3}\right), 2 \leqq p<3<q \leqq \infty$. Then

$$
\lim _{\lambda \rightarrow \pm \infty}\left\|F\left(H_{0}(\theta, R)-\lambda \mp i\right)^{-1} G\right\|=0
$$

uniformly with respect to $\theta \in \overline{\mathbb{C}}_{a}^{ \pm}$.

Proof. We write:

$$
F\left(H_{0}(\theta, R)-\lambda \mp i\right)^{-1} G=\mp i \int_{0}^{\infty} F e^{\mp i t H_{0}(\theta, R) \pm i \lambda t-t} G d t .
$$

Let us now decompose the integral in the right hand side of (2.44) as

$$
\int_{0}^{\varepsilon} F \exp \left(\mp i t H_{0}(\theta, R) \pm i \lambda t-t\right) G d t+\int_{\varepsilon}^{\infty} F \exp \left(\mp i t H_{0}(\theta, R) \pm i \lambda t-t\right) G d t
$$

Using Corollary 2.8, it is enough to show that for any $\varepsilon>0$ the second term vanishes in norm as $|\lambda| \rightarrow \infty$ uniformly with respect to $\theta$. By a standard approximation argument, we may assume $f, g \in C_{0}^{\infty}\left(\mathbb{R}^{3}\right)$.

In this case a simple computation shows that

$$
\sup _{\theta \in \overline{\mathbb{C}}_{\mathrm{a}}^{ \pm}}\left\|F \exp \left(\mp i t H_{0}(\theta, R) \pm i \lambda t-t\right) G\right\|_{W^{1,1}(\varepsilon \leqq t<\infty ; B(\mathscr{H}))}<+\infty .
$$

Since we can regard the integral as a Riemann integral in $B(\mathscr{H})$, by the RiemannLebesgue lemma, we have (2.43).

2.10. Lemma. (1) Let $V \in L^{2}+L_{\varepsilon}^{\infty}$. Then for any $\theta \in \mathbb{C}_{a}, V\left(H_{0}(\theta, R)-z\right)^{-1}$, $z \notin \sigma\left(H_{0}(\theta, R)\right)$, is a compact-operator valued analytic function of $z$.

(2) Let $V=V_{1}+V_{2}$ be such that $\operatorname{supp} V_{1} \subset \Omega_{-}, V_{1} \in L^{q}, 3<q \leqq \infty$, and $V_{2} \in L^{\infty}$ with $\left|V_{2}(x)\right| \rightarrow 0$ as $|x| \rightarrow \infty$. Then for $\theta \in \mathbb{C}_{a}^{ \pm}, \quad V\left(H_{0}(\theta, R)-\lambda \mp i\right)^{-1} V$ and 
$V\left(H_{0}(\theta, R)-\lambda \mp i\right)^{-1}$ are analytic in $\theta$ and

$$
\begin{aligned}
& \lim _{\lambda \rightarrow \pm \infty}\left\|V\left(H_{0}(\theta, R)-\lambda \mp i\right)^{-1}\right\|=\lim _{\lambda \rightarrow \pm \infty}\left\|V\left(H_{0}(\theta, R)-\lambda \mp i\right)^{-1} V\right\|=0, \\
& \sup _{\theta \in \mathbb{C}_{\mathbf{a}}^{+}} \sup _{\lambda \in \mathbb{R}}\left(\left\|V\left(H_{0}(\theta, R)-\lambda \mp i\right)^{-1} V\right\|+\left\|V\left(H_{0}(\theta, R)-\lambda \mp i\right)^{-1}\right\|\right)<\infty .
\end{aligned}
$$

Proof. (1) Since $D\left(H_{0}(\theta, R)\right) \subset H^{2}\left(\Omega_{-}\right) \oplus H^{2}\left(\Omega_{+}\right)$, and the image of the unit ball in $H^{2}\left(\Omega_{ \pm}\right)$under $V$ is a compact subset of $L^{2}\left(\Omega_{ \pm}\right)$, the first statement is obvious.

(2) Let us first assume $V_{2}$ has compact support. In this case the second $=$ of (2.45) follows from Corollary 2.9. When $\theta \in \mathbb{R}, H_{0}(\theta, R)$ is self-adjoint and

$$
\begin{aligned}
& \left\|V\left(H_{0}(\theta, R)-\lambda \mp i\right)^{-1}\right\|^{2} \\
& \quad \leqq \frac{1}{2}\left\{\left\|V\left(H_{0}(\theta, R)-\lambda-i\right)^{-1} V\right\|+\left\|V\left(H_{0}(\theta, R)-\lambda+i\right)^{-1} V\right\|\right\} \rightarrow 0 .
\end{aligned}
$$

When $\operatorname{Im} \theta=\eta>0$,

$$
V\left(H_{0}(\theta, R)-\lambda-i\right)^{-1}=V_{1} G_{\theta}^{-+}(\lambda+i)+V_{1} G_{\theta}^{--}(\lambda+i)+V_{2}\left(H_{0}(\theta, R)-\lambda-i\right)^{-1},
$$

and

$$
\left\|V_{2}\left(H_{0}(\theta, R)-\lambda-i\right)^{-1}\right\| \leqq\left\|V_{2}\right\|_{\infty}, \quad\left\|V_{1} G_{\theta}^{--}(\lambda+i)\right\| \leqq\left\|V_{1} G_{\theta}^{--}(0)\right\|<+\infty .
$$

Furthermore with $\theta=\xi+i a$, we have by (2.26)-2.28):

$$
\begin{aligned}
& \left\|V_{1} G_{\theta}^{-+}(\lambda+i)\right\|^{2} \leqq\left\|V_{1} G_{\theta}^{-+}(\lambda+i)\right\|_{\text {H.S. }}^{2} \\
& \leqq C \int_{|x| \leqq R} d x \int_{e^{\xi}(|y|-R)>M} d y \\
& \cdot \frac{e^{\xi}\left|V_{1}(x)\right|^{2} \exp \left(-\cos 2 a \sin (a-\varepsilon)\left|x-R \hat{y}-e^{\xi}(|y|-R) \hat{y}\right|^{2}\right)\left(R \hat{y}+e^{\xi}(|y|-R) \hat{y}\right)^{2}}{\cos 2 a\left|x-R \hat{y}-e^{\xi}(|y|-R) \hat{y}\right|^{2}|y|^{2}} \\
& +C \int_{|x| \leqq R} \int_{0 \leqq e^{\xi}(|y|-R)<M} \frac{\left|V_{1}(x)\right|^{2}\left|R \hat{y}+e^{\xi}(|y|-R) \hat{y}\right|^{2} d y}{\cos 2 a\left|x-R \hat{y}-e^{\xi}(|y|-R) \hat{y}\right|^{2} y^{2}} \\
& \leqq C\left\{\int_{|x| \leqq R} d x \int_{|y| \geqq R} d y \frac{\left|V_{1}(x)\right|^{2} \exp \left(-C_{b}|x-y|^{2}\right)}{\cos 2 a|x-y|^{2}}+\int_{|x| \leqq R} d x \int_{R \leqq|y| \leqq R+M} d y \frac{\left|V_{1}(x)\right|^{2}}{|x-y|^{2}}\right\} \\
& <+\infty \text {. }
\end{aligned}
$$

Thus we have

$$
\sup _{\operatorname{Im} \theta=0 \text { or } a} \sup _{\lambda \in \mathbb{R}}\left\|V\left(H_{0}(\theta, R)-\lambda-i\right)^{-1}\right\|<+\infty .
$$

By this and (2.45), when $\theta \in \mathbb{R}$, we have (2.45) for any $\theta \in \mathbb{C}_{a}$ by Hadamard's three lines theorem (see e.g. [7], Sect. IX.4), since $V\left(H_{0}(\theta, R)-\lambda-i\right)^{-1}$ is clearly analytic in $\theta$ for $0 \leqq \operatorname{Im} \theta \leqq a$. To obtain (2.45), (2.46) for general $V$, we split $V=\bar{V}_{1}+\bar{V}_{2}$ with $\bar{V}_{1}$ of compact support and $\left\|\bar{V}_{2}\right\|_{\infty}<\varepsilon$ for any $\varepsilon>0$, and apply the result for potentials of compact support.

Finally we prove the analyticity in $\theta$ of $V\left(H_{0}(\theta, R)-\lambda-i\right)^{-1} V$. By Proposition 2.4, it is enough to show that $V_{1} G_{\theta}^{-+}(\lambda+i) V_{2}$ and $V_{2} G_{\theta}^{+-}(\lambda+i) V_{1}$ are analytic. By (2.47) and an analogous estimate for $\left\|G_{\theta}^{+-}(\lambda+i) V_{1}\right\|$, it suffices to show that, for $f \in C_{0}^{\infty}\left(\Omega_{+}\right)$and $f_{-} \in C_{0}^{\infty}\left(\Omega_{-}\right),\left\langle V_{1} G_{\theta}^{+-}(\lambda+i) V_{1} f_{-}, f_{+}\right\rangle$and 
$\left\langle V_{1} G_{\theta}^{-+}(\lambda+i) V_{2} f_{+}, f_{-}\right\rangle$are analytic, and this can be proved as in Proposition 2.4.

Remark. 1. As the proof clearly implies, if we assume only $V \in L^{2}+L^{\infty}$ in (1) and $V_{2} \in L^{\infty}$ in (2) without requiring $V_{2}(x) \rightarrow 0$ as $|x| \rightarrow \infty$, the same results in Lemma 2.10 remain true except the compactness. This extends the estimates in the proof of Proposition 2.4.

2. The severe condition $V_{1} \in L^{q}, 3<q \leqq \infty$ in Lemma 2.10 is required to obtain estimates which are uniform in $\theta \in \overline{\mathbb{C}}_{a}^{ \pm}$.

2.11. Lemma. Let $\theta \in \mathbb{C}_{a}^{ \pm}$and $V \in L^{p}$ with $2 \leqq p$ and $\operatorname{supp} V \subset \Omega_{-}$. Then there exists $a$ constant $C_{p, \theta, R}$ such that for $t>0$,

$$
\left\|V \exp \left(\mp i t H_{0}(\theta, R)\right)\right\| \leqq C_{p, \theta, R}\left(|t|^{-3 / 2 p}+|t|^{-3 / p}\right)\|V\|_{p} .
$$

Proof. We prove the lemma for the upper signs only. Since $\operatorname{supp} V \subset \Omega_{-}$, $V \exp \left(-i t H_{0}(\theta, R)\right)=V F_{\theta}^{--}(t)+V F_{\theta}^{-+}(t)$. Since $\chi_{-} \in L^{q}$ for any $q$, we have by Corollary 2.8 that

$$
\left\|V F^{--}(t)\right\|=\left\|V F^{--}(t) \chi_{-}\right\|=\left\|V \exp \left(-i t H_{0}\right) \chi_{-}\right\| \leqq C_{p}\|V\|_{p}|t|^{-3 / p}
$$

By (2.23), we see that

$$
\left\|F^{-+}(t) f\right\|_{2} \leqq\|f\|_{2}
$$

and

$$
\left\|F^{-+}(t) f\right\|_{\infty} \leqq C_{\theta, R} t^{-3 / 2}\left\|\exp \left(-C_{\theta, R}^{\prime} y^{2} / t\right)\right\|_{2}\|f\|_{2} \leqq C_{\theta, R}^{\prime \prime} t^{-3 / 4}\|f\|_{2} .
$$

Interpolating (2.51) and (2.52), we have $\left\|V F^{-+}(t)\right\| \leqq C_{\theta, R} t^{-3 / 2 p}\|V\|_{p}$. This and (2.50) obviously imply (2.49).

\section{Unitary Propagators and Coupled Hamiltonians}

Let us denote by $V(t, x, \theta, \mu)$ the exterior complex scaled potential with translation:

$$
V(t, x, \theta, \mu)= \begin{cases}V\left(x+\mu \omega^{-2}(\cos \omega t) E\right), & |x| \leqq R, \\ V\left(R \hat{x}+e^{\theta}(|x|-R) \hat{x}+\mu \omega^{-2}(\cos \omega t) E\right), & |x| \geqq R .\end{cases}
$$

In this and next section $0<\omega$ is fixed and $R>0$ is taken in such a way that $R>2 \omega^{-2}$. The positive branch of the square root is taken for $\theta \in \mathbb{R}$. Note that for $f, g \in D\left(H_{0}(\theta, R)\right) \subset H^{1}\left(\Omega_{-}\right) \oplus H^{1}\left(\Omega_{+}\right),\langle V(t, x, \theta, \mu) f, g\rangle_{L^{2}}$ is a $C^{1}$ function of $t$. Thus, applying Kyšinski's theorem [4], we have the following result: write

$$
\mathscr{H}_{1}(\theta) \equiv D(Q(\theta)) \subset H^{1}\left(\Omega_{-}\right) \oplus H^{1}\left(\Omega_{+}\right),
$$

and let $\mathscr{H}_{-1}(\theta)$ be its dual space, $\mathscr{H}_{1}(\theta) \subset L^{2} \subset \mathscr{H}_{-1}(\theta)=\mathscr{H}_{1}(\theta)^{*} . V(t, \theta, \mu)$ is the multiplication operator by $V(t, x, \theta, \mu)$, and $H(t, \theta, \mu)=H_{0}(\theta, R)+V(t, \theta, \mu)$ with domain $D\left(H_{0}(\theta, R)\right)$.

Clearly $H(t, \theta, \mu)$ is self-adjoint for $\theta \in \mathbb{R}$.

3.1. Theorem. The equation

$$
i \frac{\partial u}{\partial t}=H(t, \theta, \mu), \quad \theta \in \mathbb{R}
$$


generates a propagator $\{U(t, s ; \theta, \mu)\}$ enjoying the following properties:

(1) $U(t, s ; \theta, \mu)$ is a unitary operator and is strongly continuous in $(t, s)$.

(2) $U(t, t ; \theta, \mu=\mathbb{1}, U(t, s ; \theta, \mu) U(s, r ; \theta, \mu)=U(t, r ; \theta, \mu)$ and $U(t+2 \pi / \omega, s+2 \pi / \omega$; $\theta, \mu)=U(t, s ; \theta, \mu)$.

(3) $U(t, s ; \theta, \mu) \mathscr{H}_{1}(\theta) \subset \mathscr{H}_{1}(\theta)$ and, for $f \in \mathscr{H}_{1}(\theta), U(t, s ; \theta, \mu) f$ is strongly differentiable in $(t, s)$ as an $\mathscr{H}_{-1}(\theta)$-valued function. Moreover

$$
\begin{gathered}
i \frac{\partial}{\partial t} U(t, s ; \theta, \mu) f=H(t, \theta, \mu) U(t, s ; \theta, \mu) f, \\
-i \frac{\partial}{\partial s} U(t, s ; \theta, \mu) f=U(t, s ; \theta, \mu) H(s, \theta, \mu) f .
\end{gathered}
$$

(4) $\hat{S}\left(\theta^{\prime}\right) U(t, s ; \theta, \mu) \hat{S}\left(\theta^{\prime}\right)^{-1}=U\left(t, s ; \theta+\theta^{\prime}, \mu\right), \theta^{\prime} \in \mathbb{R}$.

For the proof of this theorem, see Kyšinski [4] or Simon [8]. The statement (4) follows from the uniqueness of the propagator.

According to the program discussed in the introduction, we now wish to analyze the spectrum of the Floquet operator through the operator defined on a new Hilbert space $\mathscr{K}=L^{2}\left(T_{\omega}\right) \otimes L^{2}\left(\mathbb{R}^{3}\right), T_{\omega}=\mathbb{R} /(2 \pi / \omega) \mathbb{Z}$. For $\theta \in \overline{\mathbb{C}}_{a}^{ \pm}$and $\sigma \geqq 0$, we define a one-parameter family of operators $\left\{\mathscr{U}_{0}(\sigma, \theta)\right\}$ on $\mathscr{K}$ by

$$
\left(\mathscr{U}_{0}(\sigma, \theta) f\right)(t)=\exp \left(\mp i \sigma H_{0}(\theta, R)\right) f(t-\sigma), \quad f \in \mathscr{K} .
$$

We also define, for $\theta \in \mathbb{R}, \mu \geqq 0$, and $\sigma \in \mathbb{R}$

$$
(\mathscr{U}(\sigma, \theta, \mu) f)(t)=U(t, t-\sigma, \theta, \mu) f(t-\sigma), \quad f \in \mathscr{K} .
$$

3.2. Lemma. (1) $\mathscr{U}_{0}(\sigma, \theta)$ is a strongly continuous contraction semi-group on $\mathscr{K}$. If $\theta \in \mathbb{R}$, it is a unitary group.

(2) For $\theta \in \mathbb{R},\{\mathscr{U}(\sigma, \theta, \mu), \sigma \in \mathbb{R}\}$ is a strongly continuous unitary group.

Lemma 3.2 is obvious by the definition of the operator and the properties of $\exp \left(\mp i t H_{0}(\theta, R)\right)$ and $U(t, s ; \theta, \mu)$.

We denote the generators of $\mathscr{U}_{0}(\sigma, \theta)$ and $\mathscr{U}(\sigma, \theta, \mu)$ by $K_{0}(\theta)$ and $K(\theta, \mu)$ respectively:

$$
\begin{array}{lc}
\mathscr{U}_{0}(\sigma, \theta)=\exp \left(\mp i \sigma K_{0}(\theta)\right), \theta \in \overline{\mathbb{C}}_{a}^{ \pm}, & \sigma \geqq 0, \\
\mathscr{U}(\sigma, \theta, \mu)=\exp (\mp i \sigma K(\theta, \mu)), \theta \in \mathbb{R}, & \sigma \in \mathbb{R} .
\end{array}
$$

By Lemma 3.2, $K_{0}(\theta)$ is self-adjoint for $\theta \in \mathbb{R}$ and so is $K(\theta, \mu)$; for $\theta \in \mathbb{C}_{a}^{ \pm}, \pm i K_{0}(\theta)$ is maximal accretive.

Let now $A(t, x, \theta, \mu)=V(t, x, \theta, \mu)^{1 / 2}$ with the usual choice of the branch, and let $A(\theta, \mu)$ be the multiplication operator on $\mathscr{K}$ by the function $A(t, x, \theta, \mu)$.

3.3. Lemma. Let $\mathscr{D}=H^{1}\left(T_{\omega}\right) \otimes L^{2}\left(\mathbb{R}^{3}\right) \cap L^{2}\left(T_{\omega}\right) \otimes D\left(H_{0}(\theta, R)\right)$. Then $K_{0}(\theta)$ is the closure in $\mathscr{K}$ of $-i \frac{\partial}{\partial t}+H_{0}(\theta, R)$ defined on $\mathscr{D}$. Moreover for any $\theta \in \mathbb{C}_{a}$

$$
\sigma\left(K_{0}(\theta)\right)=\bigcup_{n=-\infty}^{+\infty}\left(n \omega+e^{-2 \theta} \mathbb{R}_{+}\right)
$$


Proof. Note first that $\mathscr{D}$ is dense and $\mathscr{U}_{0}(\sigma, \theta)$ invariant. For $f \in \mathscr{D}$ we have

$$
\left.i \frac{d}{d \sigma} \mathscr{U}_{0}(\sigma, \theta) f(t)\right|_{\sigma=0}=\left(-i \frac{\partial}{\partial t}+H_{0}(\theta, R)\right) f(t) .
$$

Thus $\mathscr{D}$ is a core of $K_{0}(\theta)$ and $K_{0}(\theta)=-i \frac{\partial}{\partial t}+H_{0}(\theta, R)$ on $\mathscr{D}$. This implies the first statement.

By Fourier transforming with respect to $t$, we have $\left(-i \frac{\partial}{\partial t}+K_{0}(\theta)\right) f(t)=\mathscr{F}_{t}^{-1}(n \omega$ $+H_{0}(\theta, R)\left(\mathscr{F}_{t} f\right)(n)$ for $f \in \mathscr{D}$. Thus $K_{0}(\theta)$ is unitarily equivalent to $\bigoplus_{n=-\infty}^{+\infty}\left(n \omega+H_{0}(\theta, R)\right)$ on $\bigoplus_{n=-\infty}^{+\infty} \mathscr{H}$ and (3.7) follows from Corollary 2.5 and Proposition 2.6.

3.4. Lemma. Let $\theta \in \mathbb{C}_{a}$ and $z \notin \sigma\left(K_{0}(\theta)\right)$. Then

(1) $A(\theta, \mu)\left(K_{0}(\theta)-z\right)^{-1}$ is a compact-operator valued analytic function of $(\theta, z)$.

(2) $A(\theta, \mu)\left(K_{0}(\theta)-z\right)^{-1} A(\theta, \mu)$ is a compact-operator valued analytic function of $(\theta, z)$

(3) For any $\theta \in \overline{\mathbb{C}}_{a}^{ \pm}$,

$$
\left\|A(\theta, \mu)\left(K_{0}(\theta)-z\right)^{-1} A(\theta, \mu)\right\| \rightarrow 0 \quad \text { as } \quad \pm \operatorname{Im} z \rightarrow \infty .
$$

Proof. Let $D$ be a compact subset of $\mathbb{C}_{a}$ and $F$ a compact subset of $\bigcap_{\theta \in D} \varrho\left(K_{0}(\theta)\right)$. It is enough to prove the lemma for $(\theta, z) \in D \times F$. Since $A(\theta, \mu) B^{-1}$ and $B^{-1} A(\theta, \mu)$ are $\mathscr{B}(\mathscr{K})$-valued analytic functions of $\theta$ by $\mathrm{A} 4$, it suffices to show the lemma with $A(\theta, \mu)$ replaced by $B$. By Fourier transforming:

$$
\begin{aligned}
\mathscr{F}_{t} B\left(K_{0}(\theta)-z\right)^{-1} \mathscr{F}_{t}^{-1} & =\bigoplus_{n=-\infty}^{+\infty} B\left(H_{0}(\theta, R)+n \omega-z\right)^{-1}, \\
\mathscr{F}_{t} B\left(K_{0}(\theta)-z\right)^{-1} \mathscr{F}_{t}{ }^{-1} & =\bigoplus_{n=-\infty}^{+\infty} B\left(H_{0}(\theta, R)+n \omega-z\right)^{-1} B .
\end{aligned}
$$

By Lemma 2.10 and the resolvent equation, we see that $\left\|B\left(H_{0}(\theta, R)+n \omega-z\right)^{-1}\right\|$ and $\left\|B\left(H_{0}(\theta, R)+n \omega-z\right)^{-1} B\right\|$ are uniformly bounded in $\theta \in D, z \in F, n \in \mathbb{Z}$. Since each term $B\left(H_{0}(\theta, R)+n \omega-z\right)^{-1}$ and $B\left(H_{0}(\theta, R)+n \omega-z\right)^{-1} B$ is analytic as it was shown in Lemma 2.10, we have the analyticity of the right hand side of (3.9) and (3.10). Furthermore since both $\left\|B\left(H_{0}(\theta, R)+n \omega \mp i\right)^{-1}\right\|$ and $\left\|B\left(H_{0}(\theta, R)+n \omega \mp i\right)^{-1}\right\|$ converge to zero as $n \rightarrow \pm \infty$ for any fixed $z$, the left hand side of (3.9) and (3.10) are compact operators. This proves (1) and (2).

Statement (3) can be proved as in Lemma 2.10 since $\sup \| B\left(H_{0}(\theta, R)\right.$ $+n \omega-z)^{-1} B \| \rightarrow 0$, as $\pm \operatorname{Im} z \rightarrow \infty$ by a formula analogous to (2.44). We omit the details.

For a later use we present a stronger version of Lemma 3.4 for $\theta \in \mathbb{R}$.

3.5. Lemma. Suppose that for $j=1,2 W_{j}(t, \cdot)$ (respectively $\left.X_{j}(t, \cdot)\right)$ is an $L^{p}\left(\Omega_{-}\right)$valued (respectively $L^{\infty}\left(\Omega_{+}\right.$)-valued) bounded function of $t \in T_{\omega}$ with $3<p$. Let $Y_{j}$ be the multiplication operator by the function $W_{j}(t, x)+X_{j}(t, x)$. Then for each $\theta \in \mathbb{C}_{a}^{ \pm}$, 
$z \in \mathbb{C}^{ \pm}$and $R>0, Y_{1}\left(K_{0}(\theta)-z\right)^{-1} Y_{2}$ and $Y_{1}\left(K_{0}(\theta)-z\right)^{-1}$ are bounded operators on $\mathscr{K}$, and there exists a constant $C_{p}$ independent of $W_{j}$ and $X_{j}$ such that

$$
\begin{gathered}
\left\|Y_{1}\left(K_{0}(\theta)-z\right)^{-1}\right\| \leqq C_{p}\left(\sup _{t}\left\|W_{1}(t, \cdot)\right\|_{p}+\sup _{t}\left\|X_{1}(t, \cdot)\right\|_{\infty}\right) \equiv C_{p} \ell_{1, p}, \\
\left\|Y_{1}\left(K_{0}(\theta)-z\right)^{-1} Y_{2}\right\| \leqq C_{p} \ell_{1, p} \ell_{2, p} .
\end{gathered}
$$

Here, of course, $W_{j}(t, x)+X_{j}(t, x)$ is the function which is equal to $W_{j}(t, x)$ for $x \in \Omega_{-}$ and $X_{j}(t, x)$ for $x \in \Omega_{+}$.

Proof. Since $1=\chi_{-}(x)+\chi_{+}(x)$ and $\chi_{-} \in L^{q}$ for any $0<q \leqq \infty$, it suffices to prove the estimate (3.12). By the Laplace transform we have for $f \in C^{\infty}\left(T_{\omega}, C_{0}^{\infty}\left(\mathbb{R}^{3}\right)\right)$, writing as $Y_{j}(t)$ etc., the multiplication operators by the functions $Y_{j}(t, x)$ etc.,

$$
\begin{aligned}
& \left(Y_{1}\left(K_{0}(\theta)-z\right)^{-1} Y_{2} f\right)(t)=i \int_{0}^{\infty}\left(Y_{1} e^{-i \sigma K_{0}(\theta)+i \sigma z} Y_{2} f\right)(t) d \sigma \\
& =i \int_{0}^{\infty} Y_{1}(t) e^{i \sigma z-i \sigma H_{0}(\theta, R)} Y_{2}(t-\sigma) f(t-\sigma) d \sigma \quad\left(z \in \mathbb{C}^{+}, \theta \in \mathbb{C}_{a}^{+}\right) .
\end{aligned}
$$

Hence by Corollary 2.8, Lemma 2.11 and its adjoint statement, we have

$$
\begin{aligned}
\left\|Y_{1}\left(K_{0}(\theta)-z\right)^{-1} Y_{2} f\right\|_{\mathscr{K}}^{2}= & \int_{0}^{2 \pi / \omega} d t\left\|\int_{0}^{\infty} Y_{1}(t) e^{i \sigma z-i \sigma H_{0}(\theta, R)} Y_{2}(t-\theta) f(t-\theta) d \sigma\right\|^{2} \\
\leqq & C_{p} \ell_{1, p} \ell_{2, p} \int_{0}^{2 \pi / \omega} d t\left\{\int_{0}^{\infty} e^{-\sigma|\operatorname{Im} z|} \sigma^{-3 / p}\|f(t-\sigma)\| d \sigma\right\}^{2} \\
\leqq & C_{p} \ell_{1, p} \ell_{2, p} \int_{0}^{\pi / \omega} d t\left\{\int_{0}^{\infty} e^{-\sigma|\operatorname{Im} z|} \sigma^{-3 / p}\|f(t-\sigma)\|^{2} d \sigma\right\} \\
& \cdot\left\{\int_{0}^{\infty} e^{-\sigma|\operatorname{Im} z|} \sigma^{-3 / p} d \sigma\right\} \\
= & C_{p} \ell_{1, p^{\ell}}{ }_{2, p}\left\{\int_{0}^{\infty} e^{-\sigma|\operatorname{Im} z|} \sigma^{-3 / p} d \sigma\right\}^{2}\|f\|_{\mathscr{K}}^{2} .
\end{aligned}
$$

By the density argument this completes the proof of the lemma for the upper signs. For the lower signs the proof is similar.

By Lemma 3.4, and by the analytic Fredholm theorem, the function

$$
\left(1+A(\theta, \mu)\left(K_{0}(\theta)-z\right)^{-1} A(\theta, \mu)\right)^{-1} \equiv Q(\theta, \mu, z)
$$

is a meromorphic function of $\theta$ and $z$ (separately) taking values in $B(\mathscr{K})$. Then by the proof of Lemma 3.3 of Yajima [13], we have the following result whose proof we omit because the argument is exactly the same:

3.6. Lemma. Let $\theta \in \mathbb{R}$. Then for $\operatorname{Im} z \neq 0$ :

$$
(K(\theta, \mu)-z)^{-1}=\left(K_{0}(\theta)-z\right)^{-1}-\left(K_{0}(\theta)-z\right)^{-1} A(\theta, \mu) Q(\theta, \mu, z) A(\theta, \mu)\left(K_{0}(\theta)-z\right)^{-1} .
$$

Now as a $\mathscr{B}(\mathscr{K})$-valued function, the right hand side of (3.15) has a meromorphic continuation to $\overline{\mathbb{C}}_{a}^{ \pm}$and $z \notin \sigma\left(K_{0}(\theta)\right)$. We denote by $L(\theta, \mu, z)$ the right hand side of (3.15), continued in this way. 
3.7. Lemma. Let $L(\theta, \mu, z)$ be as above. Then

(1) $L(\theta, \mu, z)-L\left(\theta, \mu, z^{\prime}\right)=\left(z-z^{\prime}\right) L(\theta, \mu, z) L\left(\theta, \mu, z^{\prime}\right)$.

(2) $\operatorname{Ker} L(\theta, \mu, z)=\{0\}$ if $z$ is not a pole of $Q(\theta, \mu, z)$.

Proof. (1) can be shown as in Proposition 2.6. We omit the details.

(2) Let $L(\theta, \mu, z) f=0$, and write $A(\theta, \mu)\left(K_{0}(\theta)-z\right)^{-1} f=g$.

By the definition of $L(\theta, \mu, z)$, we see that

$$
0=g-A(\theta, \mu)\left(K_{0}(\theta)-z\right)^{-1} A(\theta, \mu) Q(\theta, \mu, z) g=Q(\theta, \mu, z) g .
$$

Since $z$ is not at the pole, this implies $g=0$, which in turn implies $\left(K_{0}(\theta)-z\right)^{-1} f=0$ by $(3.15): L(\theta, \mu, z) f=0$. Therefore $f=0$.

By Lemma 3.6, we find that for any $\theta \in \overline{\mathbb{C}}_{a}^{ \pm}$and $\mu \geqq 0$, there exists a closed operator, denoted again by $K(\theta, \mu)$, such that

$$
(K(\theta, \mu)-z)^{-1}=L(\theta, \mu, z), \theta \in \overline{\mathbb{C}}_{a}^{ \pm}, z \in \varrho(K(\theta, \mu)) .
$$

3.8. Corollary. $(K(\theta, \mu)-z)^{-1}$ is a meromorphic function of $\theta \in \overline{\mathbb{C}}_{a}^{ \pm}$and $z \in \varrho\left(K_{0}(\theta)\right)$.

Formally we see that $K(\theta, \mu)=-i \frac{\partial}{\partial t}+H_{0}(\theta, R)+V(\theta, \mu)$.

To conclude this section we recall a result which connects the spectral properties of the Floquet operator $U\left(\frac{2 \pi}{\omega}+s, s ; \theta, \mu\right)$ and of $K(\theta, \mu), \theta \in \mathbb{R}$.

3.9. Lemma. Let $\theta \in \mathbb{R}$ and let $\mathscr{U}_{s}(\theta, \mu)$ be the operator in $\mathscr{K}$ defined as

$$
\mathscr{U}_{s}(\theta, \mu) f(t)=U(t, s ; \theta, \mu) f(t), \quad s \leqq t<s+\frac{2 \pi}{\omega},
$$

extended by periodicity to all t. $\mathscr{U}_{\mathbf{s}}(\theta, \mu)$ is a unitary operator in $\mathscr{K}$ and

(1) $\mathscr{U}_{s}(\theta, \mu)\left(1 \otimes U\left(s+\frac{2 \pi}{\omega}, s ; \theta, \mu\right)\right) \mathscr{U}_{s}(\theta, \mu)^{-1}=\exp \left(-i K(\theta, \mu)\left(\frac{2 \pi}{\omega}\right)\right)$.

(2) If $f \in \mathscr{K}$ is an eigenvector of $K(\theta, \mu)$ with eigenvalue $\lambda: K(\theta, \mu) f=\lambda f$, then $f$ is an $\mathscr{H}$-valued continuous function of $t \in T_{\omega}$, and

$$
U(t, s ; \theta, \mu) f(s)=e^{-i \lambda(t-s)} f(t), \quad t \in \mathbb{R} .
$$

(3) If $\phi \in \mathscr{H}$ is an eigenvector of $U\left(s+\frac{2 \pi}{\omega}, s, \theta, \mu\right)$ with eigenvalue $\exp \left(-i \lambda\left(\frac{2 \pi}{\omega}\right)\right)$, then

$$
f(t)=e^{i \lambda(t-s)} U(t, s ; \theta, \mu) \phi, \quad-\infty<t<\infty,
$$

belongs to $D(K(\theta, \mu))$ and is an eigenvector of $K(\theta, \mu)$ with eigenvalue $\lambda$ :

$$
K(\theta, \mu) f=\lambda f .
$$

Since the proof is identical to Proposition 2.9 of [12], we omit it here. 


\section{Existence of Resonances}

Let us now investigate the spectrum of $K(\theta, \mu)$ for $\theta \in \mathbb{C}_{a}^{ \pm}$by perturbation theory. We first locate the spectrum of $K(\theta, 0)$. To this end, we need the following lemma. Let us denote

$$
W(x, \theta)=V(t, x, \theta, 0)= \begin{cases}V(x), & |x| \leqq R, \\ V\left(R \hat{x}+e^{\theta}(|x|-R) \hat{x}\right), & |x| \geqq R,\end{cases}
$$

and let $W(\theta)$ be the multiplication operator by $W(x, \theta)$ in $\mathscr{H}$.

4.1. Lemma. Let $W(\theta)$ be as above, and let $H(\theta)=H_{0}(\theta, R)+W(\theta)$ with domain $D\left(H_{0}(\theta, R)\right)$. Then

(1) The operator family $H(\theta)$ is a self-adjoint holomorphic family of $m$-sectorial operators.

(2) $\sigma_{\text {ess }}(H(\theta))=\sigma_{\text {ess }}\left(H_{0}(\theta, R)\right)=e^{-2 \theta} \mathbb{R}_{+}, \quad \sigma_{d}(H(\theta))=\sigma(H(\theta)) \backslash \sigma_{\text {ess }}(H(\theta))$ is contained in the union of $\sigma_{d}(H(\theta)) \cap \mathbb{R}=\sigma_{d}(H)$ and $\{z \in \mathbb{C}: \mp 2 \operatorname{Im} \theta< \pm \arg z<0\}$, $\left(\theta \in \mathbb{C}_{a}^{ \pm} ; H=-\Delta+V\right)$.

(3) $\sigma_{d}(H(\theta))$ is independent of $(\theta, R)$ and $\sigma_{\text {sing }}(H(\theta))=\emptyset$.

(4) Let $\phi, \psi$ be exterior dilation analytic vectors (i.e. $\hat{S}(\theta) \phi$ and $\hat{S}(\theta) \psi$ are the restriction to $\theta \in \mathbb{R}$ of analytic vector-valued functions of $\theta \in \mathbb{C}_{a}$ ). Then the scalar product

$$
f_{\phi, \psi}(z)=\left\langle\phi,(H-z)^{-1} \psi\right\rangle
$$

which is a priori meromorphic in $0<\arg z<2 \pi$, has a meromorphic continuation to the sector $-2 a<\arg z<2 \pi+2 a$. The set of the poles $\left\{z: f_{\phi, \psi}(z)\right.$ has a pole at $z$ for some $(\phi, \psi)\}$ coincides with $\sigma_{d}(H(\theta))$.

Proof. Since the arguments are very close to the Aguilar-Balslev-Combes ones (see e.g. [7, Sect. XIII.10]) we shall be very sketchy. Writing

$$
\begin{aligned}
&(H(\theta)-z)^{-1}=\left(H_{0}(\theta)-z\right)^{-1}-\left(H_{0}(\theta)-z\right)^{-1} W^{1 / 2}(\theta)\left(1+W^{1 / 2}(\theta)\right. \\
&\left.\cdot\left(H_{0}(\theta)-z\right)^{-1} W^{1 / 2}(\theta)\right)^{-1} W^{1 / 2}(\theta)\left(H_{0}(\theta)-z\right)^{-1},
\end{aligned}
$$

by Proposition 2.4, Proposition 2.6, and Lemma 2.10, we see that $(H(\theta)-z)^{-1}$ is a holomorphic family. Since the second term in the right hand side of (4.3) is a compact operator, (2) follows from Weyl's theorem and the Aguilar-BalslevCombes argument. Statements (3) and (4) are also consequences of standard complex scaling arguments. We omit the details.

4.2. Lemma. Let $K(\theta, \mu)$ be defined as in Sect. 3 , and let $K(\theta)=K(\theta, 0)$. Then:

(1) $K(\theta)$ is a holomorphic family of operators for $\theta \in \mathbb{C}_{a}^{ \pm}$.

(2) $K(\theta)$ is a unitarily equivalent to the direct sum

$$
\bigoplus_{n=-\infty}^{+\infty}(n \omega+H(\theta)) \quad \text { on } \bigoplus_{n=-\infty}^{+\infty} \mathscr{H} .
$$

(3) $\sigma_{\text {ess }}(K(\theta))=\bigcup_{n=-\infty}^{+\infty}\left(n \omega+e^{-2 \theta} \mathbb{R}_{+}\right)$;

$$
\sigma_{d}(K(\theta))=\bigcup_{n=-\infty}^{+\infty}\left\{n \omega+\sigma_{d}(H(\theta))\right\}
$$

$\sigma_{d}(K(\theta))$ is independent of $\theta$. 
Proof. By Corollary 3.7, $K(\theta)$ is a holomorphic family in $\theta \in \mathbb{C}_{a}^{ \pm}$, since $\mathbb{C}^{ \pm} \subset \bigcap_{\theta \in \mathbb{C}^{ \pm}} \varrho\left(K_{0}(\theta)\right)$.

This proves (1). By Fourier transforming and regarding $\ell^{2}(\mathbb{Z}) \otimes \mathscr{H}=\bigoplus_{n=-\infty}^{+\infty} \mathscr{H}$, we see by (3.15) that

$$
\begin{aligned}
(K(\theta)-z)^{-1} \cong & \bigoplus_{n=-\infty}^{+\infty}\left(\left(H_{0}(\theta, R)+n \omega-z\right)^{-1}-\left(H_{0}(\theta, R)+n \omega-z\right)^{-1} W(\theta)^{1 / 2}\right. \\
& \cdot\left(1+W(\theta)^{1 / 2}\left(H_{0}(\theta, R)+n \omega-z\right)^{-1} W(\theta)^{1 / 2}\right)^{-1} \\
& \left.\cdot W(\theta)^{1 / 2}\left(H_{0}(\theta, R)+n \omega-z\right)^{-1}\right) \\
= & \bigoplus_{n=-\infty}^{+\infty}(H(\theta)+n \omega-z)^{-1}, \quad z \in \mathbb{C}^{ \pm}, \theta \in \mathbb{C}_{a}^{ \pm}
\end{aligned}
$$

This proves (2). By (4.4) and the fact that, for any $z \notin e^{-2 \theta} \mathbb{R}_{+}$,

$$
\begin{gathered}
\|\left(H_{0}(\theta, R)+n \omega-z\right)^{-1} W(\theta)^{1 / 2}\left(1+W(\theta)^{1 / 2}\left(H_{0}(\theta, R)+n \omega-z\right)^{-1} W(\theta)^{1 / 2}\right)^{-1} W(\theta)^{1 / 2} \\
\left(H_{0}(\theta, R)+n \omega-z\right)^{-1} \| \rightarrow 0,
\end{gathered}
$$

as $n \rightarrow \infty$ by Lemma 2.10 , we see that

$$
\sigma(K(\theta))=\bigcup_{n=-\infty}^{+\infty}(n \omega+\sigma(H(\theta)) .
$$

Statement (3) obviously follows from (4.5).

It is not difficult to locate the essential spectrum of $K(\theta, \mu)$.

4.3. Theorem. Let $\theta \in \mathbb{C}_{a}$. Then for any $0 \leqq \mu \leqq 1$,

$$
\sigma_{\mathrm{ess}}(K(\theta, \mu))=\bigcup_{n=-\infty}^{+\infty}\left\{n \omega+e^{-2 \theta} \mathbb{R}_{+}\right\}
$$

Proof. This follows from Lemma 3.4, (3.15) and Weyl's theorem.

4.4. Theorem. Let $0 \leqq \mu \leqq 1$. Then

(1) $s-\lim (K(\xi \pm i \varepsilon, \mu)-z)^{-1}=(K(\xi, \mu)-z)^{-1}, \xi \in \mathbb{R}, z \in \mathbb{C}^{ \pm}$.

(2) If $\phi, \psi$ are $\mathbb{1} \otimes \hat{S}(\theta)$ analytic vectors in $\mathscr{K}$, then the scalar products

$$
f_{\phi, \psi}(z)=\left\langle(K(0, \mu)-z)^{-1} \phi, \psi\right\rangle,
$$

which are holomorphic functions of $z$ in $\mathbb{C}^{ \pm}$can be continued respectively from the upper and lower half-plane into the other one as meromorphic functions of $z$ in $\mathbb{C} \backslash\left\{\bigcup_{n=-\infty}^{+\infty}\left(e^{-2 \theta} \mathbb{R}_{+}+n \omega\right)\right\}$ (for each fixed $\left.\theta\right)$.

Proof. By the equation

$$
\left(K(\theta, \mu)-z+z^{\prime}\right)^{-1}=\sum_{n=0}^{\infty}\left(z-z^{\prime}\right)^{n}\left(K(\theta, \mu)-z^{\prime}\right)^{-n-1},
$$

and a connectedness argument, it is enough to show (1) for $z= \pm i \eta, \eta>0$ large enough. We prove it for the + case only. By (3.9), (3.10), Lemma 2.10 and 
Proposition 2.4, we see that $B(x)\left(K_{0}(\theta)-z\right)^{-1}, B(x)\left(K_{0}(\theta)-z\right)^{-1} B(x)$, as well as $\left(K_{0}(\theta)-z\right)^{-1}$ are strongly continuous as $\theta \rightarrow \xi(z=i \eta)$. Therefore by (3.15) and the obvious remark that $A(\theta, \mu) B^{-1}$ and $B^{-1} A(\theta, \mu)$ are strongly continuous we have (1).

To prove (2), we write : $\phi_{\theta}=(\mathbb{1} \otimes \hat{S}(\theta)) \phi, \psi_{\theta}=(\mathbb{1} \otimes \hat{S}(\theta)) \psi$, and

$$
f_{\phi, \psi}(z, \theta)=\left\langle(K(\theta, \mu)-z)^{-1} \phi_{\theta}, \psi_{\theta}\right\rangle, \quad \theta \in \mathbb{C}_{a}^{ \pm}, z \in \mathbb{C}^{ \pm} .
$$

For $z \in \mathbb{C}^{ \pm}$, this is an analytic function of $\theta \in \mathbb{C}_{a}^{ \pm}$, and by (1) we have $f_{\phi, \psi}(z, \theta) \rightarrow f_{\phi, \psi}(z, \xi)$ as $\theta \rightarrow \xi \in \mathbb{R}$. Since $\hat{S}(\xi), \xi \in \mathbb{R}$, is unitary, $f_{\phi, \psi}(z, \theta)$ is $\theta$-independent : $f_{\phi, \psi}(z, \theta)=f_{\phi, \psi}(z)$. Now the right hand side of (4.7) can be continued into the complement of the lower half-plane with respect to $\bigcup_{n=-\infty}^{+\infty}\left\{n \omega+e^{-2 \theta} \mathbb{R}_{+}\right\}$as a meromorphic function of $z$. (Note that, as a function of $z$, the choice of the branch depends on $\theta$.)

To prove the final theorem of this section, we recall that, by Assumption A3(2), for any $\theta \in \mathbb{C}_{a}$ we have:

$$
\lim _{\mu \rightarrow 0}\left\|(A(t, x, \theta, \mu)-A(t, x, \theta, 0)) \cdot B(x)^{-1}\right\|_{L^{\infty}\left(T_{\omega} x \mathbb{R}^{3}\right)}=0 .
$$

Hence, repeating the proof of Lemma 3.4, we obtain

4.5. Lemma. Let $\theta \in \mathbb{C}_{a}^{ \pm}$. Then for any $z \in \mathbb{C}^{ \pm}$

$$
\lim _{\mu \rightarrow 0}\left\|(K(\theta, \mu)-z)^{-1}-(K(\theta, 0)-z)^{-1}\right\|=0 .
$$

Proof. By the proof of Lemma 3.4, we have, as $\mu \rightarrow 0$ :

$$
\begin{aligned}
& \left\|A(\theta, \mu)\left(K_{0}(\theta)-z\right)^{-1}-A(\theta, 0)\left(K_{0}(\theta)-z\right)^{-1}\right\| \\
& \quad \leqq\left\|(A(\theta, \mu)-A(\theta, 0)) B(x)^{-1}\right\|_{\infty}\left\|B\left(K_{0}(\theta)-z\right)^{-1}\right\| \rightarrow 0,
\end{aligned}
$$

and similarly

$$
\left\|A(\theta, \mu)\left(K_{0}(\theta)-z\right)^{-1} A(\theta, \mu)-A(\theta, 0)\left(K_{0}(\theta)-z\right)^{-1} A(\theta, 0)\right\| \rightarrow 0 .
$$

Combining this with (3.15) we have the lemma.

4.6. Theorem. Let $\theta \in \mathbb{C}_{a}^{+}$. Then there is $\bar{\mu}>0$ such that for all $\mu<\bar{\mu}$,

(1) $\sigma_{d}(K(\theta, \mu))$ is a discrete subset of $\mathbb{C} \backslash \bigcup_{n=-\infty}^{+\infty}\left\{n \omega+e^{-2 \theta} \mathbb{R}_{+}\right\}$with possible accumulation points $\{n \omega, n \in \mathbb{Z}\}$.

(2) $\sigma_{d}(K(\theta, \mu))$ is $\theta$-invariant.

(3) $\sigma_{d}(K(\sigma, \mu)) \neq \emptyset$. More precisely, if $\lambda$ is an eigenvalue of $H$ with multiplicity $m(\lambda)$, let $\lambda+n_{1} \omega, \ldots, \lambda+n_{k} \omega$ be the eigenvalues of $H$ which differ from $\lambda$ by integer multiplies of $\omega$, and let $m_{1}(\lambda), \ldots, m_{k}(\lambda)$ be their multiplicities. Let $N(\lambda)$ $=m_{1}(\lambda)+\ldots+m_{k}(\lambda)+m(\lambda)$.

Then for all $\mu<\bar{\mu}$ there are exactly $N(\lambda)$ eigenvalues $\lambda_{1}(\mu), \ldots, \lambda_{N}(\mu)$ (counted according their algebraic multiplicity) of $K(\theta, \mu)$, such that

$$
\lim _{\mu \rightarrow 0} \lambda_{k}(\mu)=\lambda, \quad k=1, \ldots, N .
$$


The proof is a direct consequence of standard arguments of perturbation theory given the results obtained above and is therefore omitted.

\section{Resonances and Perturbation Theory}

Our purpose in this section is the justification, to the lowest non-vanishing order in perturbation theory, of the Fermi Golden Rule for the resonance width under the following additional condition on the potentials which is satisfied also by the Coulomb one.

A5. For some $\theta \in \mathbb{C}_{a}$ the following properties are satisfied:

(i) There exist $3<p \leqq \infty$ and $\varrho>1 / 3$ such that for $|\mu| \leqq \Gamma$,

$$
\begin{gathered}
\left\|\chi_{-}(x)(V(x+\mu E)-V(x))^{1 / 2}\right\|_{L^{p}\left(\Omega_{-}\right)} \leqq C \mu^{\varrho}, \\
\left\|\chi_{+}(x)(V(S(\theta, R) x+\mu E)-V(S(\theta, R) x))^{1 / 2}\right\|_{L^{\infty}\left(\Omega_{+}\right)} \leqq C \mu^{\varrho} .
\end{gathered}
$$

(ii) There exist $6 / 5<q$ and $\kappa>1$ such that for $|\mu| \leqq \Gamma$,

$$
\begin{gathered}
\left(\partial V / \partial x_{1}\right)(S(\theta, R) x) \in L^{q}\left(\Omega_{-}\right) \cap L^{\infty}\left(\Omega_{+}\right), \\
\|Z(\theta, \mu, x)\|_{L^{q}\left(\Omega_{-}\right)}+\|Z(\theta, \mu, x)\|_{L^{\infty}\left(\Omega_{+}\right)} \leqq C \mu^{\kappa},
\end{gathered}
$$

where $Z(\theta, \mu, x)=V(S(\theta, R) x+\mu E)-V(S(\theta, R) x)-\mu\left(\partial V / \partial x_{1}\right)(S(\theta, R) x)$.

It is not hard to check that the Coulomb potential $V(x)=Z /|x|$ satisfies the assumption A5 with any $3<p<9 / 2$ with $\varrho=(6-p) / p$ and $6 / 5<q<3 / 2$ with $\kappa=\frac{3}{p}-1$.

Under this condition we can prove the following theorem. To avoid unnecessary complications, we henceforth assume, as in [12], $m(\lambda)=1$ in Theorem 4.6 so that $N(\lambda)=1$ for almost every $\omega$.

5.1. Theorem. Let $H=-\Delta+V(x), E(\xi)$ its spectral measure, $H=\int \xi d E(\xi)$, $\phi$ the eigenfunction corresponding to the simple eigenvalue $\lambda, H \phi=\lambda \phi$. Let $\theta \in \mathbb{C}_{a}^{ \pm}$and $\lambda(\mu)$ be the simple eigenvalue of $K(\theta, \mu)$ such that $\lim _{\mu \rightarrow 0} \lambda(\mu)=\lambda$. Then there exists $\varepsilon>0$ such that

$$
\operatorname{Im} \lambda(\mu)=\left.\mp \pi \mu^{2}(d / d \xi)\left\langle E(\xi)\left(\partial V / \partial x_{1}\right) \phi,\left(\partial V / \partial x_{1}\right) \phi\right\rangle\right|_{\xi=\lambda+\omega}+O\left(\mu^{2+\varepsilon}\right) .
$$

To establish (5.1), let us first collect some preliminary estimates under the form of lemmas. Hereafter the parameter $\theta \in \mathbb{C}_{a}^{ \pm}$is the one taken in A5, and we assume $\theta \in \mathbb{C}_{a}^{+}$taken and fixed. We denote by $V(\theta), T(\theta, \mu), V(\theta)^{1 / 2}$, and $T(\theta, \mu)^{1 / 2}$ the multiplication operators by $V(S(\theta, R) x), T(\theta, \mu, x)=V\left(S(\theta, R) x+\mu \omega^{-2} \cos \omega t E\right)$ $-V(S(\theta, R) x), V(S(\theta, R) x)^{1 / 2}$, and $T(\theta, \mu, x)^{1 / 2}$, respectively.

5.2. Lemma. Let $F$ be a compact subset of $\varrho\left(K_{0}(\theta)\right)$. Then there exists a constant $C>0$ such that for $z \in F$,

$$
\begin{gathered}
\left\|T(\theta, \mu)^{1 / 2}\left(K_{0}(\theta)-z\right)^{-1} T(\theta, \mu)^{1 / 2}\right\| \leqq C \mu^{2 \varrho}, \\
\left\|T(\theta, \mu)^{1 / 2}\left(K_{0}(\theta)-z\right)^{-1} V(\theta)^{1 / 2}\right\| \leqq C \mu^{\varrho} \\
\left\|T(\theta, \mu)^{1 / 2}\left(K_{0}(\theta)-z\right)^{-1}\right\| \leqq C \mu^{\varrho}
\end{gathered}
$$


Proof. These estimates are obvious by Lemma 3.5, A5 and the resolvent equation.

5.3. Lemma. Let $F$ be a compact subset of $\varrho(K(\theta, 0))$. Then for all sufficiently small $|\mu|, F$ is also contained in $\varrho(K(\theta, \mu))$. Furthermore setting $R(\theta, \mu, z)=(K(\theta, \mu)-z)^{-1}$ and $R_{0}(\theta, z)=\left(K_{0}(\theta)-z\right)^{-1}$, we have

$$
\begin{aligned}
R(\theta, \mu, z)= & R(\theta, 0, z)-R(\theta, 0, z) T(\theta, \mu)^{1 / 2} \cdot T(\theta, \mu)^{1 / 2} R(\theta, 0, z) \\
& +R(\theta, 0, z) T(\theta, \mu)^{1 / 2} \cdot T(\theta, \mu)^{1 / 2} R(\theta, 0, z) T(\theta, \mu)^{1 / 2} \cdot T(\theta, \mu)^{1 / 2} R(\theta, 0, z) \\
& +R_{2}(\theta, \mu, z),
\end{aligned}
$$

with $\left\|R_{2}(\theta, \mu, z)\right\| \leqq C \mu^{2+\varepsilon}$ with some $\varepsilon>0$ for all $z \in F$.

Proof. By Lemma 3.5 and Lemma 3.6, we can write the following identities valid in $\mathscr{B}(\mathscr{K})$ :

$$
\begin{aligned}
R(\theta, 0, z)=R_{0}(\theta, z)- & R_{0}(\theta, z) V(\theta)^{1 / 2}\left(1+V(\theta)^{1 / 2} R_{0}(\theta, z) V(\theta)^{1 / 2}\right)^{-1} V(\theta)^{1 / 2} R_{0}(\theta, z) \\
R(\theta, \mu, z)= & R_{0}(\theta, z)-R_{0}(\theta, z) V(\theta, \mu)^{1 / 2} \\
& \cdot\left(1+V(\theta, \mu)^{1 / 2} R_{0}(\theta, z) V(\theta, \mu)^{1 / 2}\right)^{-1} V(\theta, \mu)^{1 / 2} R_{0}(\theta, z)
\end{aligned}
$$

where $V(\theta, \mu)$ is the multiplication operator by the function

$$
V\left(S(\theta, R) x+\mu \omega^{-2} \cos \omega t E\right) .
$$

By Lemma 5.2, $T(\theta, \mu)^{1 / 2} R_{0}(\theta, z) T(\theta, \mu)^{1 / 2}, T(\theta, \mu)^{1 / 2} R_{0}(\theta, z)$, and $R_{0}(\theta, z) T(\theta, \mu)^{1 / 2}$ are bounded operators which satisfy (5.2)-(5.4). Hence by comparing the Neumann expansions at large $\operatorname{Im} z$, once more as an identity in $\mathscr{B}(\mathscr{K})$, we have

$$
\begin{aligned}
R(\theta, \mu, z)= & R(\theta, 0, z)-R(\theta, 0, z) T(\theta, \mu)^{1 / 2} \\
& \cdot\left(1+T(\theta, \mu)^{1 / 2} R(\theta, 0, z) T(\theta, \mu)^{1 / 2}\right)^{-1} T(\theta, \mu) R(\theta, 0, z) .
\end{aligned}
$$

(This identity can be proved first for bounded $V$, then the general case may be proved by an approximation argument.) Hence, through the second order geometric expansion we obtain (5.5) with

$$
\begin{aligned}
R_{2}(\theta, \mu, z)= & R(\theta, 0, z) T(\theta, \mu)^{1 / 2}\left(T(\theta, \mu)^{1 / 2} R(\theta, 0, z) T(\theta, \mu)^{1 / 2}\right)^{2} \\
& \cdot\left(1+T(\theta, \mu)^{1 / 2} R(\theta, 0, z) T(\theta, \mu)^{1 / 2}\right)^{-1} T(\theta, \mu)^{1 / 2} R(\theta, 0, z) .
\end{aligned}
$$

By Lemma 5.2 and (5.6), this completes the proof with $2+\varepsilon=6 \varrho$.

Proof of Theorem 5.1. Let $\phi(\theta)=\hat{S}(\theta, R) \phi$. Then under the present conditions, we know that $\phi(\theta): \mathbb{C}_{a} \rightarrow L^{2}$ is holomorphic and that $H(\theta, R) \phi(\theta)=\lambda \phi(\theta), H(\theta, R)$ $=H_{0}(\theta, R)+V(\theta)$. We can of course regard $\phi(\theta)$ as a vector-valued holomorphic function in $\mathscr{K}$, with $K(\theta, 0) \phi(\theta)=\lambda \phi(\theta)$. Let $P(\theta, \mu)$ be the projection operator on the eigenspace of $K(\theta, \mu)$ associated with $\lambda(\mu)$. Then by [1, p. 77],

$$
(K(\theta, \mu)-\lambda) P(\theta, \mu)=-(2 \pi i)^{-1} \int_{\Gamma}(z-\lambda) R(\theta, \mu, z) d z,
$$

where $\Gamma=\{z:|z-\lambda|=\eta\}, 0<\eta<d, d$ being the isolation distance of $\lambda$. Then by 
Lemma 5.3, denoting by $S(\theta)$ the reduced resolvent of $K(\theta, 0)$ at $z=\lambda$, we have

$$
\begin{aligned}
\lambda(\mu)-\lambda= & \operatorname{Tr}(K(\theta, \mu)-\lambda) P(\theta, \mu)=-\operatorname{Tr}[P(\theta, \mu) T(\theta, \mu) P(\theta, \mu)] \\
& -\operatorname{Tr}[P(\theta, \mu) T(\theta, \mu) S(\theta) T(\theta, \mu) P(\theta, \mu)+S(\theta) T(\theta, \mu) P(\theta, \mu) T(\theta, \mu) P(\theta, \mu) \\
& +P(\theta, \mu) T(\theta, \mu) P(\theta, \mu) T(\theta, \mu) S(\theta)]+O\left(\mu^{2+\varepsilon}\right) .
\end{aligned}
$$

Now $S(\theta) P(\theta, \mu)=P(\theta, \mu) S(\theta)=0$, and hence $\operatorname{Tr}[S(\theta) T(\theta, \mu) P(\theta, 0) T(\theta, \mu) P(\theta, 0)$ $+P(\theta, 0) T(\theta, \mu) P(\theta, 0) T(\theta, \mu) S(\theta)]=0$. Therefore, since $P(\theta, 0) \phi(\theta)=\phi(\theta), P(\theta, 0)^{*}$ $=P(\bar{\theta}, 0)$, and $T(\theta, \mu)^{*}=T(\bar{\theta}, \mu)$, we have

$$
\lambda(\mu)-\lambda=\langle T(\theta, \mu) \phi(\theta), \phi(\bar{\theta})\rangle-\langle S(\theta) T(\theta, \mu) \phi(\theta), T(\bar{\theta}, \mu) \phi(\bar{\theta})\rangle+O\left(\mu^{2+\varepsilon}\right),
$$

which with (5.2) and (5.6) implies

$$
\operatorname{Im} \lambda(\mu)=\operatorname{Im}\langle S(\theta) T(\theta, \mu) \phi(\theta), T(\bar{\theta}, \mu) \phi(\bar{\theta})\rangle+O\left(\mu^{2+\varepsilon}\right),
$$

because the first term in the right hand side of (5.10) is $\theta$-independent and hence real. Now we expand

$$
\left.T(\theta, \mu, x)=\mu \omega^{-2} \cos \omega t \cdot\left(\partial V / \partial x_{1}\right)(S(\theta, R) x)\right)+Z\left(\theta, \mu \omega^{-2} \cos \omega t, x\right) .
$$

Since $\phi(\theta) \in D(H(\theta, R)) \subset H^{2}\left(\Omega_{-}\right) \oplus H^{2}\left(\Omega_{+}\right) \subset L\left(\Omega_{-}\right) \oplus H^{2}\left(\Omega_{+}\right)$, and the assumption A5 implies $\|T(\theta, \mu, x)\|_{L^{\left.q_{(} \Omega_{-}\right)}}+\|T(\theta, \mu, x)\|_{L^{\infty}\left(\Omega_{+}\right)} \leqq C \mu$ as well, Lemma 3.5 implies

$$
\begin{aligned}
\langle S(\theta) & T(\theta, \mu) \phi(\theta), T(\bar{\theta}, \mu) \phi(\bar{\theta})\rangle \\
= & \left(\mu / \omega^{2}\right)^{2}\left\langle S(\theta)\left(\partial V / \partial x_{1}\right)(S(\theta, R) x) \phi(\theta),\left(\partial V / \partial x_{1}\right)(S(\bar{\theta}, R) x) \phi(\bar{\theta}) \cdot \cos ^{2} \omega t\right\rangle \\
& +\left\langle S(\theta) T(\theta, \mu) \phi(\theta), Z\left(\bar{\theta}, \mu \omega^{-2} \cos \omega t, x\right) \phi(\bar{\theta})\right\rangle \\
& +\left\langle S(\theta) Z\left(\theta, \mu \omega^{-2} \cos \omega t, x\right) \phi(\theta), T(\bar{\theta}, \mu) \phi(\bar{\theta})\right\rangle \\
& +\left\langle S(\theta) Z\left(\theta, \mu \omega^{-2} \cos \omega t, x\right) \phi(\theta), Z\left(\bar{\theta}, \mu \omega^{-2} \cos \omega t, x\right) \phi(\bar{\theta})\right\rangle \\
= & \left(\mu / \omega^{2}\right)^{2}\left\langle S(\theta)\left(\partial V / \partial x_{1}\right)(S(\theta, R) x) \phi(\theta),\left(\partial V / \partial x_{1}\right)(S(\bar{\theta}, R) x) \phi(\bar{\theta}) \cos ^{2} \omega t\right\rangle \\
& +O\left(\mu^{1+\kappa}\right)+O\left(\mu^{1+\kappa}\right)+O\left(\mu^{2 \kappa}\right),
\end{aligned}
$$

whence, by the $\theta$-independence of the inner product implied by the exterior dilation analyticity, performing the integration over $t$ and repeating Simon's standard computation ([7, Sect. XII.6]; see also [12] for further details), we obtain $\operatorname{Im} \lambda(\mu)=-\left.\pi\left(\mu / \omega^{2}\right)^{2}(d / d \xi)\left\langle E(\xi)\left(\partial V / \partial x_{1}\right)(x) \phi(x),\left(\partial V / \partial x_{1}\right)(x) \phi(x)\right\rangle\right|_{\xi=\lambda+\omega}+O\left(\mu^{2+\varepsilon}\right)$

with suitably small $\varepsilon>0$, which also implies $\operatorname{Im}(\lambda(\mu)<0$ for almost every $\omega$ and $\mu$ suitably small.

\section{References}

1. Kato, T.: Perturbation theory for linear operators. Berlin, Heidelberg, New York: Springer 1966

2. Kato, T.: Wave operators and similarity for some non selfadjoint operators. Math. Ann. 162, 258-276 (1966)

3. Kitada, H., Yajima, K.: A scattering theory for time-dependent long-range potentials. Duke Math. J. (to appear)

4. Kisynski, J.: Sur les operateurs de Green des problèmes de Cauchy abstraits. Stud. Math. 23, 285-328 (1964) 
5. Lanhoff, P.W., Epstein, S.T., Karplus, M.: Aspects of time-dependent perturbation theory. Rev. Mod. Phys. 44, 602-644 (1974)

6. Mizohata, S.: Partial differential equations. Cambridge: Cambridge University Press 1973

7. Reed, M., Simon, B. : Methods of modern mathematical physics, Vols. I-IV. New York: Academic Press 1972, 1975, 1979, 1978

8. Simon, B.: Quantum mechanics of Hamiltonians defined as quadratic forms. Princeton: Princeton University Press 1972

9. Simon, B. : The definition of molecular resonance curves by the method of exterior complex scaling. Phys. Lett. 71 A, 211-214 (1979)

10. Simon, B.: Exterior complex scaling and molecular resonances in the Born-Oppenheimer approximation (to appear)

11. Scully, M.O., Sargent III, M.: The concept of the photon. Phys. Today 25, 38-47 (1972)

12. Yajima, K.: Resonances for AC-Stark effect. Commun. Math. Phys. 87, 331-352 (1982)

13. Yajima, K.: Scattering theory for Schrödinger equations with potentials periodic in time. J. Math. Soc. Jpn. 29, 729-743 (1977)

Communicated by B. Simon

Received August 16, 1982; in revised form February 7, 1983 
